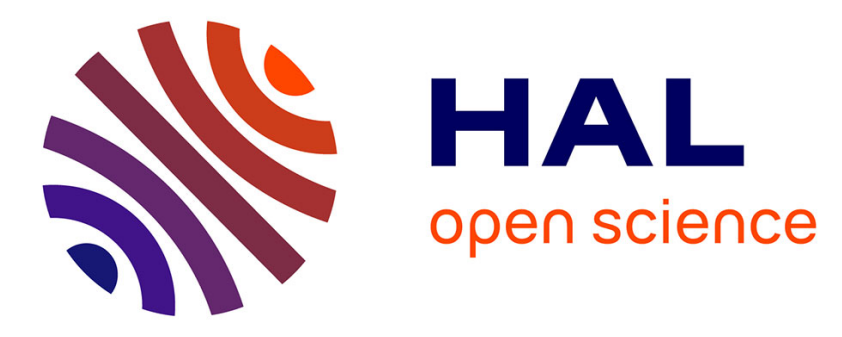

\title{
Estimation of urban zonal speed dynamics from user-activity-dependent positioning data and regional paths
}

Manon Seppecher, Ludovic Leclercq, Angelo Furno, Delphine Lejri, Thamara

Vieira da Rocha

\section{To cite this version:}

Manon Seppecher, Ludovic Leclercq, Angelo Furno, Delphine Lejri, Thamara Vieira da Rocha. Estimation of urban zonal speed dynamics from user-activity-dependent positioning data and regional paths. Transportation research. Part C, Emerging technologies, 2021, 129, pp1-28. 10.1016/j.trc.2021.103183 . hal-03461907

\section{HAL Id: hal-03461907 \\ https://hal.science/hal-03461907}

Submitted on 1 Dec 2021

HAL is a multi-disciplinary open access archive for the deposit and dissemination of scientific research documents, whether they are published or not. The documents may come from teaching and research institutions in France or abroad, or from public or private research centers.
L'archive ouverte pluridisciplinaire HAL, est destinée au dépôt et à la diffusion de documents scientifiques de niveau recherche, publiés ou non, émanant des établissements d'enseignement et de recherche français ou étrangers, des laboratoires publics ou privés. 


\title{
Estimation of Urban Zonal Speed Dynamics from User-Activity-Dependent Positioning Data and Regional Paths
}

\author{
Manon Seppecher ${ }^{\mathrm{a}, \mathrm{b}, *}$, Ludovic Leclercq ${ }^{\mathrm{b}, * *}$, Angelo Furno ${ }^{\mathrm{b}}$, Delphine Lejri ${ }^{\mathrm{b}}$, Thamara Vieira da Rocha ${ }^{\mathrm{a}}$ \\ ${ }^{a}$ CITEPA, 42 rue de Paradis, 75010 Paris, France \\ ${ }^{b}$ Univ. Gustave Eiffel, Univ. Lyon, ENTPE, LICIT, F-69518, Lyon, France
}

\begin{abstract}
Over the past few decades, the digitalization of services and infrastructures has led to the emergence of a broad set of new information sources to characterize human mobility. These sources usually offer valuable significant population penetration rates but may also suffer from important temporal sparsity. Data generated by user activity, such as social networks or mobile phone data, especially fit this description. Although this temporal sparsity might prevent estimating individual travel speeds, we state that such low-frequency positioning data enable estimating the average urban traffic speed dynamics when considering an adequate network partitioning. In this sense, this article proposes a new method, based on the division of the urban area of a given city into regions and on the analysis of a limited set of basic characteristics of individual vehicle trips, such as the regional path. Our solution first involves estimating robust travel times from travelers sharing similar trip features and then jointly analyzing these travel times to deduce the underlying regional traffic speeds, using regression analysis. We apply this methodology on a set of trips derived from a large GPS dataset of vehicle tracks covering the city of Lyon. These data are purposely downsampled to reduce the sampling rate and reproduce bias and temporal features that are proper to sparser but larger-scale, mobility data sources dependent on user's communication activities. Controlling the data downsampling process allows us to evaluate the impacts of the progressive information loss on the speed estimation, while the raw GPS data provide the ground truth speed reference against which to compare our results. Provided that the amount of observed individual trips is sufficient, the analysis returns satisfying speed estimation results, both at low and high downsampling levels. Thus, we successfully demonstrate that it is possible to estimate zonal traffic speeds from degraded trip data without evaluating individual travel speeds.
\end{abstract}

Keywords: traffic speed estimation, travel time estimation, regional paths, regions, bias model, mobile phone data, CDR data, GPS data, LBNS data

\section{Highlights}

- We propose a framework for estimating traffic speeds from temporally-biased trips.

- We propose a temporal bias model linked to user-activity-dependent positioning data.

- We apply the methodology to a set of GPS trips downsampled according to this model.

- We investigate the effect of successive data degradation on the results.

- We investigate the effect of statistical representativity on the results.

\footnotetext{
${ }^{*}$ Corresponding author. Tel.: +33 (0) 4720472 95, manon.seppecher@ univ-eiffel.fr

${ }^{* *}$ Corresponding author. Tel.: +33 (0) 4720477 16, ludovic.leclercq@ @niv-eiffel.fr
} 


\section{Introduction}

Over the last two decades, the digitalization of services and infrastructures has led to the emergence of a broad set of new information sources to characterize human mobility. In particular, GPS tracks from navigation systems and services have become prevalent (Castro et al., 2013; Lin and Hsu, 2014). The exploitation of other sources, such as anonymous geolocalized social media logs (Twitter, Foursquare) and cell phone data, has also become increasingly popular (Chen et al., 2016). The collected geolocated tracks may vary significantly in both the spatial and the temporal resolution depending on the technology used to generate the data (GPS, mobile telephony, wireless networks), the sensing device or service (on-board or mobile navigation systems, geolocation through social networks and locationbased services, 2G, 3G 4G cellular networks), as well as the level of user activity (Asgari et al., 2013; Toch et al., 2018).

GPS tracks derived from vehicular and mobile navigation systems are usually quite accurate both in space and time. The navigation system generally acquires the user's position at a regular frequency, which usually ranges from a very high frequency (e.g., every second) to lower sampling rates (in the order of the minute). Despite these possible variations and acquisition noise and errors, GPS navigation systems remain a key source to explore individual and aggregated mobility patterns and monitor traffic (Castro et al., 2013; Lin and Hsu, 2014). However, the related data sets often suffer from limited penetration rates.

Another source of information on human mobility can be found in social networks and Location-Based (Networking) Services (LBS - LBNS). For instance, the Twitter social network allows users to share their geo-location with their tweets, while the LBNS Swarm (formally called Foursquare) offers its users to "check-in" in various venues and share this information with friends. GPS being the technology on which those networks and services rely, the spatial accuracy of the data generated using such services is mostly the same as that of navigation systems. However, the main difference with the latter lies in the data generation process. Instead of being automatic and regular, the availability of geolocated samples with social networks and location-based services depends on the user's communication and sharing behaviors. In particular, users with little posting and check-in activities will generate fewer location data, and their mobility becomes harder to estimate.

This is a characteristic that social networks and location-based services data share with several types of passive mobile phone data, such as Call Detail Records (CDR) and network signaling data (which, in addition to calls and texting events, include network control ones such as handovers). These data are passively generated by mobile phone users while communicating and are collected and stored by communication data providers for billing or network management purposes. CDR data register each communication event (i.e., a call, message, or data browsing event emitted or received by a cellular device) at the base station scale (i.e., antenna), while handovers register each base station involved in a call. Thus, the less a user communicates, the fewer data will be generated. Barabási (2005), and later Candia et al. (2008); Gonzalez et al. (2008); Calabrese et al. (2011) and Chen et al. (2018), explored the existence of patterns in mobile communication behaviors and observed that the latter are bursty. While most of the users' communication events happen within short time intervals, some significant time gaps also exist between successive dense communication sequences. Interestingly, Gandica et al. (2017) demonstrated that message posting on Twitter presents similar temporal characteristics. Those results suggest that these user-activity-dependent positioning data (UADP data further on) may be more fitted to identify and analyze the static phases (often called stays) of users' routines than to characterize the trips in-between (Ranjan et al., 2012; Hoteit et al., 2017).

An extensive literature exists on the use of user-activity-dependent positioning data for mobility analysis (see Blondel et al. (2015); Naboulsi et al. (2016) on mobile phone data), but it mainly focuses on the characterization of mobility patterns rather than the analysis of dynamic traffic features. This literature is often based on methods to detect and process communication events that take place during periods of human immobility (e.g., see Jiang et al. (2013); Toole et al. (2015)), which allow inferring origin and destination locations of trips, for instance. On the basis of such methods, the subjects covered by the literature vary from the exploration of mobility habits and characteristics (Jurdak et al. (2015) with Twitter data) and the development of realistic mobility choice models (Gonzalez et al. (2008) based on CDR data) to the construction of origin-destination matrices (see Osorio-Arjona and García-Palomares (2019) with Twitter data, Iqbal et al. (2014); Çolak et al. (2015); Alexander et al. (2015) with CDR data) and their use as a proxy for the traditionally costly transportation surveys. However, when it comes to describing the trips themselves, the irregularity of the communication behaviors and the individual data generation may result in little to no positional information during trips that is therefore much harder to exploit. Even if some data are collected during a trip and can 
help to identify the likely traveled routes, as shown in Jiang et al. (2013), this situation is far from being systematic and only concerns few positions. Due to this limitation, the studies related to dynamic mobility pattern characterization are less developed. In Toole et al. (2015), a CDR-based origin-destination matrix is estimated in a first step, then assigned onto the road network in a second step to estimate the traffic load. Handovers and Location Area Updates are used to estimate traffic speeds on highway segments (Bar-Gera, 2007; Ou et al., 2011), travel time (Janecek et al., 2015), or Macroscopic Fundamental Diagrams (MFD) (Derrmann et al., 2017). However, handovers guarantee a minimum frequency of location updates during calls, which is not the case for other data sources such as traditional CDR or social media logs. Whether UADP data can still be used to derive dynamic traffic characteristics, such as speed, remains an open question.

Monitoring urban network traffic speed is crucial for many applications, including traffic control, route guidance, or emission calculations (Zhang et al., 2011); and targeting speeds from irregular and low-frequency positioning data remains the most challenging application. In fact, the traditional bottom-up speed estimation methods from GPS floating vehicle tracks (Zheng et al., 2013; Shang et al., 2014), which rely on averaging individual speeds calculated at the road segment level, cannot be transposed to this kind of data. However, user-activity-dependent positioning data have significant advantages with respect to more conventional traffic data sources. They are usually accessible and massive. Mobile phone data have very high penetration rates among the populations (Blondel et al., 2015; Algizawy et al., 2017; Bachir et al., 2017), which results in excellent spatial coverage in urban areas. Social network data are massive as well. They still offer lower penetration rates (because they correspond to more specific audiences and uses) than cell phone data, but their availability continues growing (Cisco, 2020), offering promising perspectives in more extensive use for mobility analysis. Traffic speed estimations based on GPS floating vehicle tracks often rely on complementary data sources (like surveys, loop detectors, or cameras) to implement spatial extrapolation processes and compensate for the low data coverage (Shang et al., 2014; Zhan et al., 2017), leading to costly overall processes. On the contrary, working with temporally sparse but massive data seems promising as it could offer cost-efficient and large-scale alternative methods. Given the massive amounts in which UADP data are available, and despite their temporal irregularity and sparsity, we aim to prove that they offer in an urban context a viable alternative to GPS floating car data to estimate the mean traffic speed dynamics at a zonal scale. By focusing on UADP data, we consider all massive mobility data related to the use of new technologies and whose temporal sampling frequency depends on users' communication behaviors and activities, and therefore inherently uncertain.

A key point of the method we propose is that it is based on the partition of the urban network into regions characterized by homogeneous traffic conditions. This partition defines a new spatial scale at which the individual trip data are up-scaled and analyzed. This aggregation process allows characterizing interrelated travelers, i.e., travelers who simultaneously cross the same network areas, but is also more adapted to the possible raw spatial resolution of the data than the road segment scale. Thanks to this new scale, our method only requires a set of elementary trip features but no explicit characterization of individual local speeds. Those features are the observed departure and arrival time, and the regional path (as defined in Yildirimoglu and Geroliminis (2014); Batista et al. (2019)), i.e., the succession of regions traveled by individuals between their origin and their destination regions.

We propose to fuse from the outset the travel time information of individuals traveling along the same regional paths on a periodic regular basis (e.g., every 15 minutes), and conduct, for each of the considered period, a combined analysis of the average travel times estimates derived from this data fusion. Provided that a reliable estimation of the trip lengths at the city and regional scales is available from external offline sources, this analysis allows deducing a broad and consistent estimation of the regional average traffic speeds. One of the main challenges of applying this methodology is the correct estimation of average travel times, despite the temporal biases inherent to the use of user-activity-dependent positioning data. The method we propose relies on statistical considerations to addresses this challenge.

We apply the method to a set of artificially temporally-biased trips derived from a real GPS dataset of tracked vehicles traveling in the Great Lyon area, France. This approach allows using the original GPS dataset as a groundtruth reference for traffic speed, against which to assess the methodology and determine whether the simulated data are qualified for urban traffic speed estimation. Although the GPS dataset size is limited, literature works have shown that GPS floating car data was a particularly reliable source for estimating zonal traffic speed. Contrary to other traffic variables, the traffic speed estimation does not require scaling processes. Its estimation from vehicle probe data results in very satisfactory results despite low penetration rates (Nagle and Gayah, 2014; Leclercq et al., 2014). In this research, by keeping the data downsampling process under control instead of directly using UADP data, we aim 
at better understanding how the jamming and the consequent progressive information loss could impact the quality of the results. By focusing on a synthetic data context that permits clear identification of the temporal bias, this study aims to assess the robustness of the proposed methodology towards its application on non-synthetic UADP data.

This article is organized as follows. Section 2 exposes the principle of our approach and describes the proposed methodology. Section 3 presents our case study, as well as the exploited data. Section 4 focuses on the results we reached. Finally, Section 5 concludes with the limits and perspectives of this work.

\section{Methodology}

\subsection{Problem statement}

We focus on exploiting vehicle trips extracted from a generic UADP dataset (mobile phone data, LBNS data, or any similar mobility dataset) leveraging the literature stay detection methods. These methods define stays as locations (either a specific position or a cluster of positions close to each other) where users are observed for a minimum amount of time. These methods are therefore geared towards identifying static phases of individual mobility. This paper will consider that such methods are reliable and that the stays detected do indeed correspond to static phases. Nevertheless, this identification is dependent on the communication activity of the users. It implies that static phases may only be partially identified if the user is not active at the beginning or the end of their stay. Suppose we define a trip as the mobility phase between two consecutive stays. In that case, an important distinction must be made between the observed trip departure and arrival times and the exact (but unknown) ones, as the varying communication rates of users provide sparse information on their mobility. In this paper, we use the following definitions, $i$ being an individual trip:

- The observed departure time is defined as the time when the last static event of the origin stay is observed. By definition, the observed departure time precedes the actual one. In this paper, let $\varepsilon_{d}^{i}$ be the positive bias between these two values (all mathematical notations in the article are listed in the notation table in Appendix A).

- Reciprocally, the observed arrival time is defined as the time when the first static event of the destination stay is observed. By definition, the observed arrival time follows the actual one. Let $\varepsilon_{a}^{i}$ the positive bias between these two values.

- The observed travel time $T_{\text {obs }}^{i}$ is defined as the time elapsed between two consecutive stays, i.e., between the observed departure and the observed arrival times. It is an overestimate of the actual travel time $T^{i}$.

Based on these definitions, we have:

$$
\varepsilon^{i}=T_{o b s}^{i}-T^{i}=\varepsilon_{d}^{i}+\varepsilon_{a}^{i}
$$

Intermediate trip positions can give additional information, considering that the departure time occurs between the observed departure time and the first mobile event. The reasoning is symmetrical for the arrival time. Therefore, the longer the delay between consecutive moving and static events, the more uncertain the departure and arrival times, the greater the risk of significant overestimation of the individual travel time. These individual biases are, by nature, very difficult to estimate at the trip level, and they affect the observations of the individual travel time themselves.

In this context, the problem we address in this paper is the following. Can we provide a method to correctly estimate traffic speed at least at an aggregated regional scale despite these unknown individual biases? 


\subsection{Overview}

The fundamental principle behind the speed estimation method we propose is that the overall sample size of the data can compensate for the low data quality at the individual trip level. The method relies on the fusion of individual trip information and statistical considerations to provide a reliable regional traffic speed estimation. It requires the implementation of the following steps.

1. Network partitioning and time resolution definitions;

2. Average de-biased travel time estimation through the periodic gathering of similar trips;

3. Speed calculation through the resolution of a linear system model;

\section{Speed trends smoothing.}

These steps constitute the generic skeleton of the methodological framework we propose. However, some of these steps will require adaptation to the specific properties of the input data and case study.

The network partitioning is the starting point of our methodology. It participates in the definition of the spatiotemporal resolution of the final speed results and identifying similar trips. Fine-resolution road network data constitute the primary input of such a network partitioning process. However, the spatial resolution of the analyzed UADP data determines the minimal resolution of the regional segmentation of the city. For instance, the spatial resolution of cell phone data generally corresponds to the underlying base station network. In this case, the partitioning of the urban network must result in larger regions than the Voronoi tessellation of the base station network.

The regional partitioning of the network impacts the data structure required for several inputs.

On the one hand, our method requires that the vehicle trips database (the key input of the method) include a coarse representation of the trip trajectory consistent with the previously defined scale, called a regional path. Therefore, the network partitioning affects this feature of vehicle trips. The other trip features are the observed arrival time and observed travel time. This minimal travel data structure corresponds to a generic intermediate format that is reasonably accessible by preprocessing the raw UADP data, regardless of their specific characteristics. The implementation details of this preprocessing step depend on these specific characteristics. They are not addressed in this paper to preserve the generality of our framework. However, in Section 5, we shed light on the challenges linked to this step and provide options to overcome them.

On the other hand, the regional partitioning also constraints the average trip length estimates matrix, a critical input for the speed calculation phase. This matrix records local average trip lengths according to different macroscopic itineraries. Section 2.3 provides more details on its structure. This matrix is computed once, offline, and before the trip data analysis. This calculation can be based on the analysis of GPS data, if available, as done in this study. Those data must have sufficient coverage to calculate statistically reliable distances. However, alternatives exist, such as methods that exploit travel surveys or the automatic and systematic analysis of the road network topography (Batista et al., 2019).

Finally, the travel time estimation step requires an accurate evaluation of the average travel time bias caused by uneven user activity patterns. This evaluation, which relies on an analysis of the specific UADP data, is considered to be an input of the method. It will allow the observed travel times to be de-skewed and the average travel times to be estimated correctly.

Figure 1 illustrates the succession of the methodological steps and their articulation with the different inputs cited above. The following sections describe in more detail each of these steps.

\subsection{Network partitioning and time resolution definitions}

One of the essential steps in the methodology is the identification and the fusion of similar trips. However, sparse trips distributed over space and time are difficult to compare and relate to one another. In this section, we first propose to define a new spatial and temporal scale, thus laying the ground for the definition of comparison criteria between different trips. The definition of such a new scale relies on both spatial and temporal aggregation.

We first define a new spatial scale. The targeted urban road network is partitioned into regions. These regions must mainly be characterized by homogeneous city fabric, demography, road network topology, and, most importantly, 


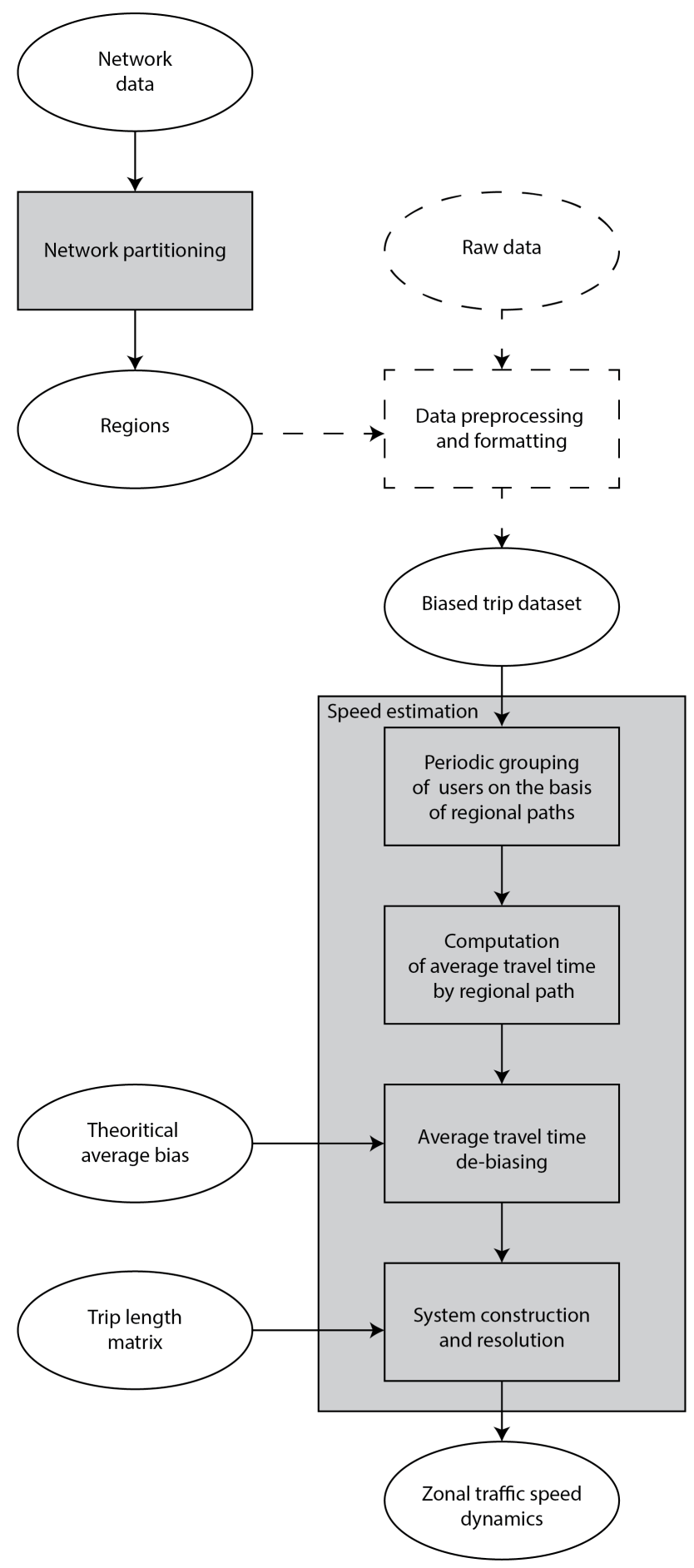

Figure 1: Methodological framework 
traffic dynamics. Homogeneity of traffic dynamics is an essential requirement for a robust estimation of the regional mean speed, as shown by the literature on the Macroscopic Fundamental Diagram (Daganzo, 2007; Geroliminis and Daganzo, 2008). Following the network partitioning guidelines provided by the related literature, one can divide a city into a set of regions usually ranging from 5 to 20 . This new spatial scale will later determine the final spatial resolution of traffic speed estimates. Therefore, it must be adapted to the precision requirements of the case study and, where appropriate, to the resolution of the data, as mentioned above. This regional scale provides the background for defining a fundamental notion of our method, the regional path:

- The regional path is defined as the sequence of the successive regions traveled from the origin to the trip destination. Therefore, it is a coarse representation of the path followed at the road segment scale, consistent with the regional partitioning of the network.

This up-scaling process is illustrated in Figure 2. While Figure 2a displays an individual trip at the road segment network, Figure $2 \mathrm{~b}$ represents its corresponding regional path $R_{1} R_{4} R_{6}$. Further on, we will consider that trips follow the structure defined here:

- We call trip the ternary structure defined by a regional path, an observed travel time and an observed arrival time.

The trip length estimation that must be performed beforehand of the method is also constrained by the previously defined regional scale and paths. This input shall record the average regional trip length in each region along each possible regional path. Thus, it can take the shape of a distance matrix $\hat{\boldsymbol{L}}$ where rows are the different possible regional paths, and columns are the different regions resulting from the spatial tessellation. The cell value at $(i, j)$ corresponds to the average distance traveled in the $j^{t h}$ region, when traveling along the $i^{t h}$ regional path $P$. It is equal to zero if the path $P$ does not cross the $j^{\text {th }}$ region. This matrix is assumed to be constant over time, but time-dependent patterns can be considered if they can be characterized independently on another dataset (Batista et al., 2021a).

Besides the change of spatial scale, we define a new temporal resolution. The evaluation period is discretized into equal time intervals. This new temporal reference imposes the temporal granularity of the speed evaluation and must be chosen accordingly. In particular, the temporal unit must be small enough to reproduce the rapidly changing speed dynamics during peak hours. We choose 15 minutes in this study, as commonly used in the literature.

These processes of partitioning the temporal dimension and the studied network, and the resulting notion of the regional path, provide temporal and spatial criteria for comparing different paths that are otherwise difficult to compare and the basis for identifying similar trips. The following relations are defined:

- $R 1$ : Two trips that share the same regional path are called spatially similar.

- $R 2$ : Two trips that share the same (exact) arrival period are called simultaneous.

- R3: Two trips that satisfy both $R 1$ and R2, i.e., that are spatially similar and simultaneous, are called overlapping.

These rules allow establishing a comparison between individual trips. This comparison is a crucial aspect of our methodology, which relies on identifying overlapping trips and fusing their observed travel time before de-skewing it.

However, as previously anticipated, it is essential to remind the difference between the exact arrival time and the observed one (extracted from UADP data). Such travel times might correspond to different periods if the user's communication rates are low. Therefore, identifying overlapping trips theoretically requires correcting the observed biased arrival time. Nevertheless, in the two following sections, we neglect this bias and consider that the exact arrival time is known when presenting the core methodological framework. We will relax this favorable assumption in Section 2.6. 


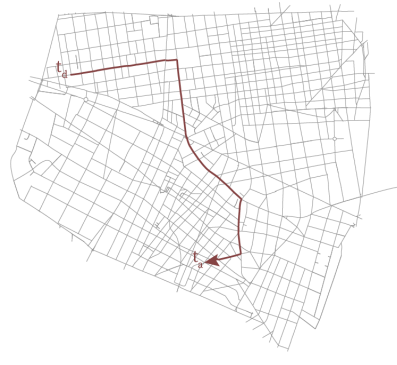

(a)

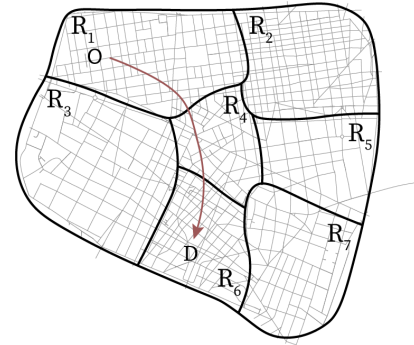

(b)

Figure 2: Representation of the different data quality for a same individual trip. (a) GPS track of an individual departing from their origin at time $t_{d}$ and arriving at time $t_{a}$. (b) The scaling up of the track at a regional scales accounts for those inaccuracies and reduce the route to a core path feature: the regional path $R_{1} R_{4} R_{6}$

\subsection{Average travel time estimation}

The robust estimation of travel times over the network is a critical milestone in our methodology. The travel time observations featured in the trip data can provide, to some extent, a snapshot of the traffic conditions that individuals encounter along their regional route at a given period. However, at an individual level, these observations are not reliable enough because they are sensitive, on the one hand, to the microscopic origin, destination, and routing of trips at the network level and, on the other hand, above all, to the frequency of individual observations.

As anticipated in the previous section, the observed travel time of a trip can be related to its exact travel time via the introduction of an additive temporal bias. Although other (non-additive) forms of bias could be considered, this model is the simplest to start with, and a priori the most natural. Let $P$ be a regional path, and let $i$ be an individual trip traveling along $P$. We thus have:

$$
T_{P, o b s}^{i}=T_{P}^{i}+\varepsilon^{i}
$$

where $T_{P, o b s}^{i}, T_{P, o b s}^{i}$ and $\varepsilon^{i}$ are, respectively, the observed travel time of $i$ along $P$, the exact travel time of $i$ along $P$, and the travel time bias of trip $i$.

Although estimating this individual bias would allow de-skewing the observed travel time, this bias is, by nature, difficult to assess. However, the estimation of its average seems less challenging and can allow to de-skew on average the observed travel times. This average bias is assumed known and to be an input of our framework. This hypothesis is discussed in Section 5. To this end, we propose merging overlapping trips and averaging their observed travel times to build a unique aggregated biased travel time information by path and period.

Let $t$ represent a generic period, and let $I_{P}^{t}$ be the set of overlapping trips along $\mathrm{P}$ that reach destination at time $t$, with $n_{t, P}=\left|I_{P}^{t}\right|$. Averaging Equation 2 over $I_{P}^{t}$ gives:

$$
\bar{T}_{P}^{t}=\bar{T}_{P, o b s}^{t}-\bar{\varepsilon}_{P}^{t}
$$

where $\bar{T}_{P}^{t}, \bar{T}_{P, o b s}^{t}$ and $\bar{\varepsilon}_{P}^{t}$ are, respectively, the average actual travel time, the average observed travel time, and the average bias of trips from cluster $I_{P}^{t}$.

Assuming that the bias is independent of the trip path and time (hypothesis $\mathrm{H}_{2}$, discussed below), we can consider that the distribution of individual biases $\varepsilon^{i}$ can be modeled via a unique random variable $X$. The construction of such a model, and the estimation of its first moment $\mu_{X} \equiv E(X)$, can offer an approximation of $\bar{\varepsilon}_{P}^{t}$ allowing the de-skewing of $\bar{T}_{P, o b s}^{t}$, provided that the sample of individuals associated with this period and path is large enough:

$$
\bar{T}_{P}^{t} \approx \bar{T}_{P, o b s}^{t}-\mu_{X}
$$

One of the great advantages of merging overlapping trips data together is that it makes the estimation of the average travel time more robust, as long as $\mu_{X}$ can be independently characterized. This trip aggregation greatly reduces the complexity of estimating travel times and traffic speed from biased temporal data, since neither the estimation of the individual biases, nor the characterization the bias distribution are needed. Only the estimation of its average value 


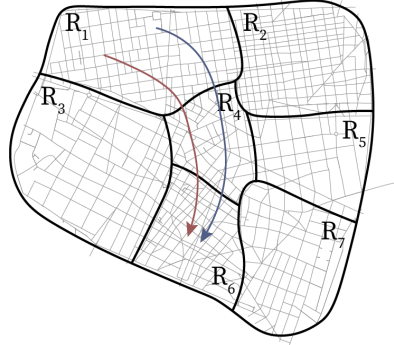

(a)

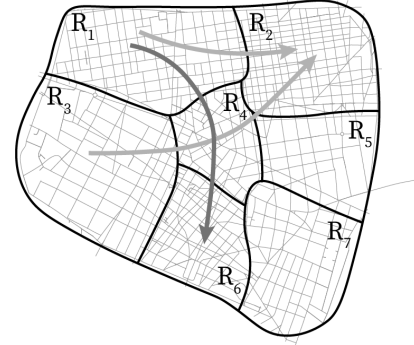

(b)

Figure 3: Visualization of the data merging into clusters of similar trips. (a) Two individuals traveling simultaneously along a same regional path despite following different (unknown) routes. (b) Merging those individuals into a unique average object (in dark grey) allows characterizing the average travel time needed to travel the regional path $R_{1} R_{4} R_{6}$. This is repeated for every regional path and helps in characterizing the travel time over the whole network.

Due to the data temporal sparsity of individual tracks, $T_{P, r}^{i}, L_{P, r}^{i}$, and $V_{r}^{i}$ are considered unknown

Although vehicles may experience different local and instantaneous speeds over an area, their average speeds depend mostly on overall traffic conditions, and mainly on the accumulation (i.e., number of vehicles in the region).

is required. However, the sampling size is a key condition of the process: the larger the sample is, the better the theoretical average bias $\mu_{X}$ is representative of the sample's average bias.

The temporal independence of the bias can be discussed in light of the work of Chen et al. (2018), who showed, using CDR data, that the inter-event time distribution is sensitive to the hour of the day and. In particular, longer inter-event times are observed during nighttime and early morning. However, these results account for all individuals, including the ones that are static and sleeping, while we are exclusively interested in moving ones. Thus, our hypothesis comes down to considering that users' communication activities are more related to their general activity level (mobile or static) than to the hour of the day, which seems reasonable. The spatial independence of the data is similarly debatable since mobile phone or social network use is correlated with socio-demographics. Thus, it would be interesting to validate or refute our hypothesis with a study of the evolution of the inter-event time of traveling users through time and space, but this goes beyond the scope of this paper. The assumption made here allows considering a first simple de-skewing approach. Future researches on the travel time bias associated with UADP data could further complete this approach by differentiating the average bias according to time or space.

The systematic estimation, for each $P$ and $t$, of the observed travel times, and their de-skewing using an average bias estimate results in a robust, spatially exhaustive, and dynamic evaluation of the travel times across the network at each period. In that sense, Figure 3 illustrates how two overlapping trips are jointly analyzed to build a unique representative object of the traffic conditions along $R_{1} R_{4} R_{6}$. Figure $3 \mathrm{~b}$ also shows how this can be repeated for every regional path of the network. The speed estimation process relies on this systematic mean regional path travel time estimation.

\subsection{Speed estimation}

This section develops the mathematical foundations of the speed estimation method.

Starting at the individual level, we consider an individual trip $i$ of $I_{P}^{t}$. Its exact traveled time $T_{P}^{i}$ along $P$ can be expressed as the sum of the traveled times $T_{P, r}^{i}$ over each region $r$ of $P$ (see Equation 5). The regional travel time terms can be in turns expressed as the fraction of the distance traveled by $i$ in $r$ (i.e., $L_{P, r}^{i}$ ) over the mean spatial speed of $i$ in region $r$ (i.e., $V_{r}^{i}$ ), as described in Equation 6.

$$
\begin{aligned}
\forall i \in I_{P}^{t}, \quad T_{P}^{i} & =\sum_{r \in P} T_{P, r}^{i} \\
T_{P}^{i} & =\sum_{r \in P} \frac{L_{P, r}^{i}}{V_{r}^{i}}
\end{aligned}
$$


These speeds show little scatter among individuals, and can be approximated by the mean spatial speed of all individuals traveling in the region. This observation has sustained the development of the MFD theory (Daganzo, 2007; Geroliminis and Daganzo, 2008). The partitioning of the network into sub-regions of consistent traffic dynamics is especially meant to enforce this assumption. On this basis, we assume that each regional speed is homogeneous and constant over the duration of each period $t$, so that:

$$
V_{r}^{i}=V_{r}^{t}, \quad \forall i
$$

where $V_{r}^{t}$ is the regional spatial mean speed at period $t$.

In Equation 6, after summing on the $I_{t, P}$ trips, this gives:

$$
\sum_{i=1}^{n_{t, P}} T_{P}^{i}=\sum_{i=1}^{n_{t, P}} \sum_{r \in P} \frac{L_{P, r}^{i}}{V_{r}^{t}}=\sum_{r \in P} \sum_{i=1}^{n_{t, P}} \frac{L_{P, r}^{i}}{V_{r}^{t}}
$$

Equation 8 can easily be rewritten as follows:

$$
\begin{aligned}
\sum_{i=1}^{n_{t, P}} T_{P}^{i} & =\sum_{r \in P} \frac{1}{V_{r}^{t}} \sum_{i=1}^{n_{t, P}} L_{P, r}^{i} \\
n_{t, P} \bar{T}_{P}^{t} & =\sum_{r \in P} n_{t, P} \frac{\bar{L}_{P, r}^{t}}{V_{r}^{t}} \\
\bar{T}_{P}^{t} & =\sum_{r \in P} \frac{\bar{L}_{P, r}^{t}}{V_{r}^{t}}
\end{aligned}
$$

Again, a significant advantage of this averaging process over the sample $I_{P}^{t}$ is that the characterization of individual regional trip lengths $L_{P, r}^{i}$ for any individual $i$ becomes unnecessary. Instead, the sample mean value $\bar{L}_{P, r}^{t}$ turns out to be sufficient. On condition that the sampling size is large enough, this can be replaced by its static estimate $\hat{L}_{P, r}$, drawn from the exogenous trip length matrix $\hat{L}$ described above:

$$
\bar{T}_{P}^{t} \approx \sum_{r \in P} \frac{\hat{L}_{P, r}}{V_{r}^{t}}
$$

At this stage, the computed distance matrix is used to express, through Equation 12, a relationship between the average travel time along path $P$ at period $t$, and the underlying, unknown traffic speeds of the regions along the $P$.

Although the average trip duration $\bar{T}_{P}^{t}$ is unknown, in Section 2.4 we discussed how a knowledge of the average time bias $\mu_{X}$ could allow to estimate it. Based on Equation 4, we thus get:

$$
\bar{T}_{P, o b s}^{t}-\mu_{X} \approx \sum_{r \in P} \frac{\hat{L}_{P, r}}{V_{r}^{t}}
$$

At each period $t$ and for each path $P$, the average travel time along $P, \bar{T}_{P, o b s}^{t}$, can be derived from the UADP analysis. Conversely, the constant distance parameters $\hat{L}_{P, r}$ are drawn from the aforementioned estimated trip length matrix $\hat{L} . \mu_{X}$ is assumed known as well. $V_{r}^{t}$ are the only unknowns of the system. When applying in Equation 13 the change of variable $x_{r}^{t}=1 / V_{r}^{t}$, we finally get the unbiased system:

$$
\forall t, \quad S^{t}=\left\{\bar{T}_{P, o b s}^{t}-\mu_{X}=\sum_{r \in P} \hat{L}_{P, r} x_{r}^{t}, \quad \forall P\right\} .
$$

In Equation 14, we name $S^{t}$ the linear system composed of $|R|$ unknowns $\left(x_{r}^{t}, r \in R\right)$ and as many equations as the number of regional paths observed during the reference period $t$. The UADP data analysis and the parameters 
extracted from the trip length matrix allow to fully characterize the system, which can be rewritten in matrix notation as:

$$
\forall t, \quad S^{t}=\left\{\boldsymbol{T}_{o b s}^{t}-\boldsymbol{\mu}_{X}=\hat{\boldsymbol{L}}^{\mid t} \boldsymbol{x}^{t}\right\}
$$

where $\boldsymbol{T}_{o b s}^{\boldsymbol{t}}$ is the average observed travel time vector and $\hat{\boldsymbol{L}}^{\mid t}$ is the sub-matrix of $\hat{\boldsymbol{L}}$ restricted to the regional paths observed at period $t$.

Given that the number of regional paths will generally exceed the number of regions of the adopted partitioning, $S^{t}$ is very likely over-determined. Consequently, the system will probably have no exact solution, but an approximated one can be calculated using regression analysis. To this purpose, we apply a non-negative least squares regression method to the system. For a given over-determined linear system $\boldsymbol{A x}=\boldsymbol{y}$, in which $\boldsymbol{A}$ is a matrix, $\boldsymbol{x}$ the unknown vector and $y$ the response one, the ordinary least square problem consists of finding the optimal $x$, which minimizes the sum of the squared residuals. This can be formulated as solving $\boldsymbol{x}_{\mathbf{0}}=\operatorname{argmin}_{\boldsymbol{x}}\|\boldsymbol{A} \boldsymbol{x}-\boldsymbol{y}\|_{2}$, with \|\|$_{2}$ the euclidean norm. Additional constraints on the elements of $x$ can be added. This is the case in the non-negative least square method, implying that the coefficient of $\boldsymbol{x}$ be non-negative. In our case, such constraint allows for taking into account the non-negative nature of zonal traffic speed. We apply the non-negative least square method to $S^{t}$, by solving at each time step the following:

$$
\boldsymbol{x}_{0}^{t}=\operatorname{argmin}_{\boldsymbol{x}}|| \hat{\boldsymbol{L}}^{\mid t} \boldsymbol{x}^{t}-\boldsymbol{T}_{\text {obs }}^{t}+\boldsymbol{\mu}_{X} \|_{2}, \quad \boldsymbol{x} \geq 0
$$

The non-negative least square method $n n l s$, implemented in Python's package Scipy, was used in this paper. Taking the reciprocal values of the solution vector $x_{0}^{t}$ gives the optimal speed vector $v_{0}^{t}$. This resolution process can be iterated throughout the whole studied time span to estimate the complete temporal speed trends. It should be noted that in this paper, the intra-region trips were filtered out of the system and discarded from the analysis, as they contribute to a diagonal subsystem whose optimization seems to take precedence over the other system equations in the regression analysis.

\subsection{Arrival time correction and data selection}

The previous sections have considered the arrival period $t$ to be exact. However, when extracting trips from UADP data, not only the travel time is biased, but so are the arrival time and period. Let $t_{0}^{i}$ be the actual precise arrival time, and $t_{0, o b s}^{i}$ the observed precise arrival time, by opposition to $t^{i}$ and $t_{o b s}^{i}$ that refer to the actual and observed arrival periods. We have:

$$
t_{0, o b s}^{i}=t_{0}^{i}+\varepsilon_{a}^{i}
$$

When reducing the temporal resolution to the period level, this implies that the observed arrival period does not necessarily corresponds to the actual arrival period. This results in the following inequality:

$$
t_{o b s}^{i} \geq t^{i}
$$

Consequently, identifying simultaneous trips based on the observed arrival period might correspond to considering together users that refer in reality to other periods, with potentially different traffic speeds. Therefore, the correct gathering of simultaneous trips ideally requires recovering for each individual their exact arrival times from their observed arrival times. This recovering cannot be done on average, as for the travel time de-skewing. Deducting the expected value of the arrival bias (i.e., half of the expected value of the travel time bias $\mu_{X}$ ) from the observed arrival times of each trip, as in Equation 19, only shifts all trips by the same amount of time, but not re-assign each trip to its correct arrival period.

$$
t_{0}^{i \prime}=t_{0, o b s}^{i}-\mu_{X} / 2
$$


While this shift may help correct an average time offset and slightly modify individuals' grouping, it can in no way correct the massive mixing of trips together. Such a correction requires the precise estimation of individual biases separately, which seems very hard to achieve considering the nature of the data. In this paper, we abandon the idea of applying such an individual bias correction and stick to correcting the average arrival time offset by considering the new arrival period $t_{0}^{i \prime}$ as defined by Equation 19. Nevertheless, to fully meet with the challenge raised by this arrival bias, we also propose to enhance at each period the robustness of the linear system by implementing filtering solutions at different levels.

Firstly, one can consider filtering individuals according to a criterion based on their communication rates, in order to limit to some extent the mixing of individual trips corresponding to different periods. In practice, the individual overall average inter-event time can be used as an indicator of these communication rates. However, this filter must be considered with caution, as it might impact the sampling size. In our study, trips are not associated with individual inter-event times but with individual biases. We will explore the impact of filtering trips based on a criterion addressing these biases.

Second, we suggest implementing a filter on the minimal number of trips to consider that an equation defining a regional path at a given period is valid. Setting this minimal threshold aims at ensuring the robustness of the travel time estimates despite potential shuffled trips. One could also consider setting a maximum threshold on the travel time standard deviation, which could be particularly suitable for large trip samples.

The criterion above focuses on the reliability of each equation independently of others. A third element we consider is the consistency of the equations with each other. As this coherence is quite challenging to evaluate, we propose a sensitive filtering approach to stabilize the results obtained from a set of indiscriminate equations. The approach we propose is based on bootstrapping, a statistical inference method based on random sampling. For a given period, for which the data processing resulted in a system $S$ made of $n$ distinct equations, a set of subsystems $S_{i}$ is generated and solved to explore the sensitivity of the results to the structure of the system. Specifically, the generic subsystem $S_{i}$ is built by sampling with replacement the same number $n$ of equations from $S$. Consequently, $S_{i}$ has the same number of $n$ equations but possible redundancy for some of them. To take this redundancy into account, we resolve the system with a weighted least square optimization method. The weight of each equation is given by its number of occurrences in the subsystem. Thus, the more an equation is sampled from the original system, the higher its weight in the resolution. This process is iterated over many subsystems (we set the minimal number of iterations to 100) to explore the results' sensitivity to different sampled equations and weighting parameters. Consequently, many derived speed solutions are generated at each period, resulting in a speed distribution for each region. We apply statistical filters to these distributions to filter out the most aberrant values before averaging the remaining speeds. This process enforces the results' consistency and stabilizes them without explicitly labeling equations as reliable or not and arbitrarily filtering them out.

Although our method does not exclude does not exclude working with a favorable sub-population displaying the lowest communication inter-event times, the individual filtering limits the reach of the method by reducing the range of users considered. Filtering individuals according to their inter-event travel time corresponds to considering a subpopulation with a reduced average bias. Therefore, among these three filtering methods, the last two are considered preferable in the evaluation of our methodology.

\subsection{Speed trends smoothing}

The speed estimation process described above is applied independently at each time step. The results of this recursive application of the method to consecutive periods may present sawtooth instabilities between consecutive periods due to variations of the regional paths observed, their number or the amount of travelers they represent. To smooth speed trends over time and ensure consistency of results between consecutive periods, we implement a dynamic filtering based on a rolling window method. The window size is set to a chosen number of periods $n$. At each period $t$, the smoothed traffic speed is calculated as :

$$
\bar{V}_{r}^{t}=\frac{1}{n} \sum_{i=0}^{n-1} V_{r}^{t-\frac{n-1}{2}+i}
$$


Because the speed trends can vary faster at peak hours than during the remaining periods of the day, we increase the sensibility of the filter at this time. Then, $n$ is set to 3 (periods) during assumed peak hours, while it is set to 5 the rest of the day.

\subsection{Discussion}

In this section, we discuss a few insights we can retrieve from the structure of the system.

First, the structure of the system $S^{t}$ directly explains the impact of the chosen tesselation. The more fine-grained the spatial resolution, the larger the system size. Not only does the number of unknown variables (regional speeds) increase, but so does the number of possible regional paths, and hence of equations. Consequently, an increase in the number of regions also leads to a relative decrease in regional paths' attendance level, as they are more numerous and therefore less crossed. This attendance decrease might be problematic as the methodology relies on the hypothesis of sufficient sample representativeness. Thus, determining the appropriate spatial partitioning raises the question of finding the suitable trade-off between a fine-grained traffic speed estimation and a system composed of reliable equations.

Additionally, the shape of the system provides an insight into the importance of the average travel time de-skewing. The speed vector resulting from the approximated resolution of the system $S^{t}$ of Equation 15 is reliable under the condition that the system is properly conditioned, i.e., that the average travel time vector $\boldsymbol{T}_{\text {obs }}^{\boldsymbol{t}}-\boldsymbol{\mu}_{X}$ is correctly estimated (the trip length matrix distance factors $\hat{\boldsymbol{L}}$ being considered as reliable). Without accounting for the average bias generated by the users' uneven activity rhythms, characterizing the regional network travel times based on the observed travel times would result in an overstated left side of the system compared to the latent actual average travel time $\bar{T}_{P}^{t}$. This system would be unrepresentative of the actual traffic speeds and likely to underestimate them.

\section{Experimental approach}

To evaluate the performance of the proposed methodology, we apply it to a UADP dataset derived from highfrequency GPS data through data simplification and downsampling. This evaluation approach, based on highfrequency raw data instead of low-frequency data, presents several advantages. First, it provides control over the average data bias, which is an essential part of the methodology that has not been enough characterized by the literature. Second, it allows exploring the impact of the data simplification, the temporal downsampling, and the de-skewing process on the speed estimation quality. This exploration is a necessary step to identify the strengths and weaknesses of the method and adjust it accordingly. It helps to understand how to increase the robustness of the method before applying it to real inaccurate and biased UADP data, for which the corresponding accurate ground truth GPS tracks will most likely be lacking. Last but not least, this experimental approach provides an easily accessible and reliable baseline estimation of the traffic speed dynamics, against which to compare our results. The original GPS dataset also provides valuable data for estimating the trip lengths, which are assumed in our framework to be derived from exogenous sources and produced offline before UADP-like data processing.

\subsection{Bias model}

In the previous section, we discussed how the temporal biases of the data could substantially impact the speed estimation results and why it was necessary to take into account this bias in order for the speed estimation system to be adequately conditioned. This led to Equation 15. To the best of our knowledge, no model is characterizing this bias. Thus, we propose a simplistic and generic bias distribution modeling to downsample GPS trips and simulate the temporal characteristics of data with low-sampling rates. The modeled bias is positive and independent both of time and space, in agreement with the considerations and assumptions developed in Section 2. The model relies on the characterization of the sample inter-event time distribution, which is a standard indicator to measure the sampling rates of user-activity-dependent positioning data. The generated bias distribution will be applied to the individual trips in order to simulate temporal biases and explore the performance of the method on these downsampled trips.

The travel time bias is modeled by a random variable $\mathrm{X}$, which we aim to characterize. To start, we express the individual travel time bias $\varepsilon^{i}$ as the sum of a departure and arrival offsets $\varepsilon_{d}^{i}$ and $\varepsilon_{a}^{i}$ (see Equation 1):

$$
\varepsilon^{i}=\varepsilon_{d}^{i}+\varepsilon_{a}^{i}
$$


$\varepsilon_{d}^{i}$ being the time difference between the actual and observed departure times from the origin stay, and $\varepsilon_{a}^{i}$ the time difference between the observed and actual arrival times at the destination stay. They are considered to be positive. Hence, if the departure and arrival temporal offsets $\varepsilon_{d}^{i}$ and $\varepsilon_{a}^{i}$ are themselves modeled by the same random variable Y, Equation 21 gives $X=2 Y$. Now, we consider that an individual's departure time from a stay position can occur with a uniform probability between the pre-departure communication event and the post-departure communication event. The delay between these two events follows the distribution of the user's inter-event times, which we assimilate with the population's inter-event times distribution for the sake of simplicity.

Mathematically, this means that departure bias follows a uniform distribution law bounded by the population's inter-event time distribution. Symmetrically, the same reasoning applies to the arrival bias.

Here, we model the population's inter-event time by a simple exponential law $Z$ of parameter $\lambda$. While the literature often reports inter-event time distribution closer to truncated power law distribution, we select an exponential distribution here out of simplicity. It requires a single parameter $\lambda$ directly linked to the distribution average.

The considerations above lead to:

$$
\begin{aligned}
Z & \sim \operatorname{Exp}(\lambda) \\
Y \mid Z & \sim U(0, z)
\end{aligned}
$$

and that the first two moments of $\mathrm{Y}$ are:

$$
E(Y)=\frac{1}{2 \lambda} \quad \text { and } \quad V(Y)=\frac{5}{12} \frac{1}{\lambda^{2}}
$$

444 for $X=2 Y$

$$
\begin{aligned}
f_{Z}(z) & =\lambda e^{-\lambda z} \\
\text { and } f_{Y \mid Z}(y \mid z) & =\left\{\begin{array}{cl}
\frac{1}{z} & 0 \leq y \leq z, \\
0 & \text { otherwise }
\end{array}\right.
\end{aligned}
$$

From this, we show (see the detailed calculation in Appendix B) that the probability density function of $Y$ is:

$$
f_{Y}(y)=\lambda \int_{0}^{+\infty} \frac{e^{-\lambda(y+z)}}{y+z} d z
$$

$$
\mu_{X} \equiv E(X)=2 E(Y)=\frac{1}{\lambda} \quad \text { and } \quad V(X)=4 V(Y)=\frac{5}{3} \frac{1}{\lambda^{2}}
$$

We already stressed the importance of the size of $I_{P}^{t}$ to ensure that $\mu_{X}$ is representative of the cluster average bias. This is all the more important as with our bias model, as the variance of $\mathrm{X}$ increases with mean inter-event time $E(Z)=\frac{1}{\lambda}$ :

$$
V(X)=\frac{5}{3} E(Z)^{2}
$$

Equation 29 shows that the larger the mean inter-event time is, the more scattered the trip bias distribution will be, and the more data per period and per regional path will be needed to ensure a reliable de-biasing process.

With this model, fairly simple and realistic, we propose a way to simulate the travel time biases related to the users' variable mobile phone activity rates. The construction of the model makes it possible to approximate the average bias 


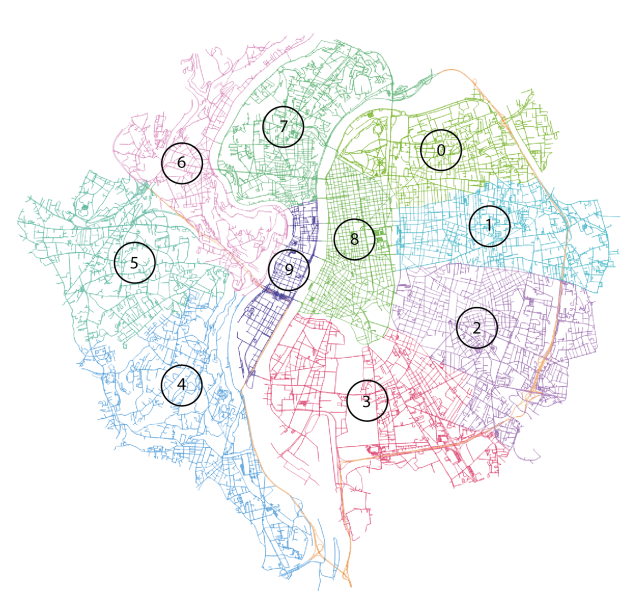

(a)

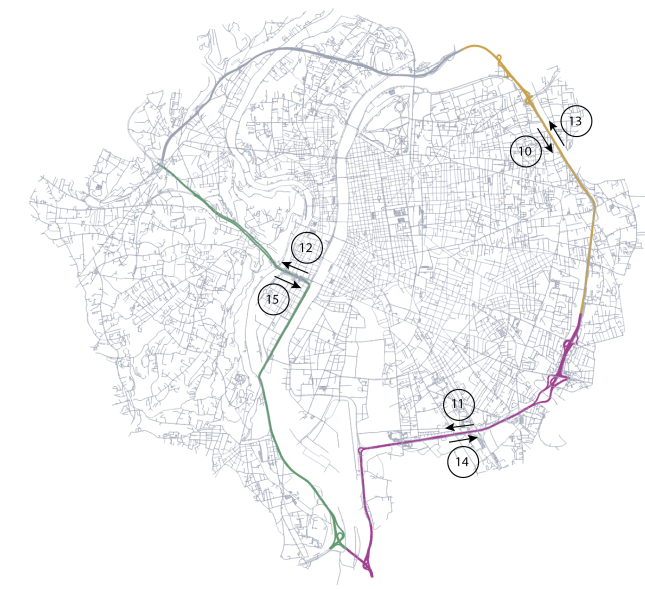

(b)

Figure 4: Maps of the regions partitioning the city of Lyon, France. (a) Map of the urban regions; (b) Map of the ring road regions. The ring road is divided into three zones, which are themselves separated into two according to the direction of traffic.

of the measured travel times from the analysis of the population inter-event time distribution and deduce it from them. While an exponential inter-event time distribution was chosen here, the method is transposable to any other observed distribution. Although this model was developed for the data simulation purposes and not validated against real UADP data, we look forward to evaluating its relevance in a real context. We also believe that the relationship between bias and inter-event time will remain a key part of a more complex bias modeling process.

\subsection{Spatial partitioning}

The city of Lyon, France, is chosen as our case study. The study area includes Lyon and the neighboring municipality of Villeurbanne, located inside Lyon's ring road. We have parted this territory into sixteen distinct regions. Ten of them divide the urban areas, while the ring road is extracted and parted into six regions, three per each direction. Those regions are displayed in Figure 4. Urban regions were manually defined based on the natural geographical barriers (two rivers) and the major road networks. The major adopted criteria consisted of separating the main arterial roads in different regions. The traffic variables were verified to be relatively uniform in each region (Mariotte et al., 2020). We split the ring road into three main blocks based on our knowledge of daily congestion patterns: the northeast, south-east, and south-west blocks. The remaining north-west section of the ring road is mainly a tunnel. As the GPS data are lacking in this section, it was ignored in the analysis. We checked whether the two opposite travel directions could be jointly considered a homogeneous traffic area by analyzing the ring road speed profiles. As the speed profiles appeared to be significantly different, we decided to split the ring road further, regions per direction. It is important to mention here that despite the efforts to ensure the homogeneity of the traffic conditions inside each zone, some aspects of the network structure can be a limitation. In particular, many motorways serve Lyon and relate it to the neighboring cities. Those motorways cross the urban regions and cause within regions traffic heterogeneity (region $0,2,3,4$, and 6). One solution to limit this heterogeneity would be to isolate those motorways sections into new specific regions. However, this would unnecessarily increase the number of traffic speed variables. Instead, we propose a light and easy-to-implement adaptation of the overall methodology to take this aspect into account. Although this filter is specific to our case study and the chosen partitioning, it can be applied again in other contexts, as cities are often served by expressways passing through peripheral residential areas.

Based on our knowledge of the traffic in Lyon, we assume that the trips traveling along those motorways are very likely to travel along the ring road as well, as a transition to another motorway or their final destination in an urban region of the city. Consequently, the ring road is assumed to be more strongly connected to these motorways than to the rest of the urban regions. Hence, we propose to decouple our estimation equation system as follows.

On one side, a first subsystem $S_{R R}^{t}$ is built from the regional paths that travel along the ring road at one point. The system is solved and returns a first speed vector $V_{0}^{t}$. The corresponding equations are assumed to carry reliable 


\begin{tabular}{llllll}
\hline & Day 1 & Day 2 & Day 3 & Day 4 & Day 5 \\
\hline Number of trips & 19597 & 20750 & 20951 & 21963 & 22302 \\
\hline
\end{tabular}

Table 1: Number of trips considered per day

information about the traffic speed on the ring road. However, the information they carry about the dynamics in the other urban regions (traveled before or after the ring road) is assumed to characterize better the traffic condition in their motorways than in their urban grid. Consequently, while the solution $V_{0}^{t}$ is considered reliable for characterizing the ring road speed, it is considered as unreliable to characterize the urban regions' speeds.

On the other side, we build a second subsystem $S_{U R B}^{t}$ with regional paths that do not travel along the ring road. The resolution of this new subsystem results in a second speed vector $V_{1}^{t}$. This solution only characterizes the urban regions and is assumed to be reliable on them.

Both solutions are merged to build a unique speed vector $V^{t}$ built from the concatenation of $V_{1 \mid \text { URB }}^{t}$ the speed vector $V_{1}^{t}$ restricted to the urban components and $V_{0 \mid R R}^{t}$ the speed vector $V_{0}^{t}$ restricted to the ring road components.

\subsection{Data description}

The GPS dataset exploited in this study consists of cleaned and map-matched GPS traces over the Greater Lyon area, i.e., an area larger than the perimeter selected for our study. A European navigation system provider collected the data between October 2017 and September 2018. The traces are collected from multiple navigation system technologies equipping a multitude of observed floating vehicles (29,000 vehicles per day on average on the Greater Lyon). Moreover, as each trace corresponds to a vehicle, there is no need to filter out pedestrian or cyclist travelers as usually required when working with mobile phone or social networks data. This aspect slightly facilitates the problem of estimating traffic speeds, since the question of detecting the mode of transport does not arise here.

The trips used in this study were extracted from five typical weekdays, i.e., from Monday, February 12, to Friday, February 16, 2018. As few trips are observed at night-time in our dataset, the time span selected for our evaluation is restrained to day-time hours, i.e., in-between 5 AM and 8 PM. The data from the full month of February 2018 was used for the offline calculation of trip lengths.

\subsection{Trip data preparation}

The first phase of data processing involves filtering and further cleaning the data. As the area covered by the GPS data is larger than the studied perimeter, we applied a first filter to remove from the data the segments of GPS tracks outside the relevant perimeter. Moreover, the GPS tracks are additionally parsed into different trips when stays are detected. Additional steps included filtering out redundant individuals, static vehicles, and GPS tracks that are fragmented or do not have a spatial consistency, to obtain a clean and reliable data set. At the end of this preprocessing step, the number of trips per considered day is as described in Table 1. Although these numbers are significant, we insist on the importance of a minimal sample size at the period and regional path level. At each time step, and for each path, the number of trips must be large enough so that the expected value of the bias is representative of the sampled biases. As GPS data are limited in sample size, we artificially extend the size of the dataset by duplicating each trip 100 times. This trick allows obtaining an extended sampled population, that is then downsampled and biased for each individuals.

This GPS trip dataset is then strictly reduced to the trip features needed by the methodology. The actual travel and actual arrival times of each trip are directly extracted from the GPS data observation. Additionally, every GPS track is down-scaled to the spatial resolution previously defined, to obtain the regional path information. Those three trip features (regional path, actual arrival time, and actual travel time) are stored, along with the trip id, in a new dataset that will be called $D S_{0}$ in the following. At this stage, a first downsampling level has been introduced in the spatial dimension to replace the precise track information with the regional path feature. Although travel times do not yet include any temporal bias at this stage, the trip representation is then already considerably simplified. This dataset will be the subject of our first experiments.

The last processing step consists of applying to $D S_{0}$ a temporal downsampling process that aims to simulate the temporal imprecisions of UADP data compared to GPS data. The idea is to simulate the travel time increase caused 


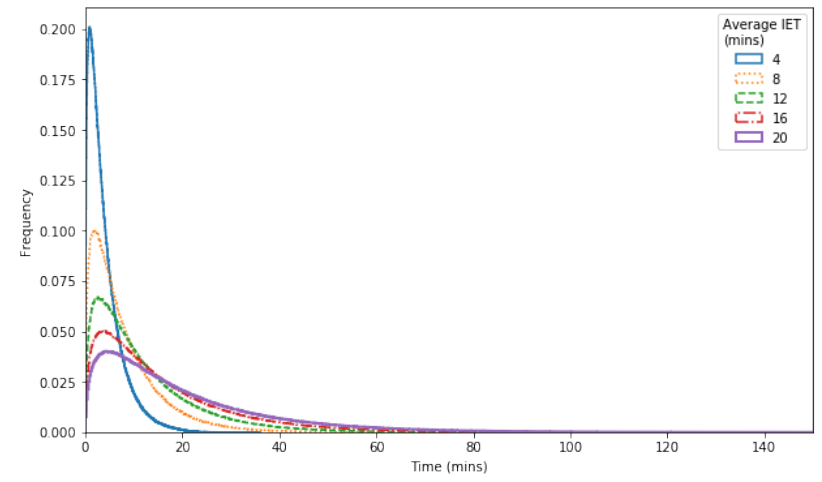

Figure 5: Bias distributions depending on the selected average inter-event time

by the temporal biases that the uneven inter-event communication times introduce in the departure and arrival time detection, using the bias model described in Section 3.1. The average inter-event time (IET) is a crucial parameter of this model. The value of this parameter may depend on the population observed or on the type of data chosen: for example, using handovers and signalization datasets will display weaker inter-event times than CDR or LBNS data. To take this inter-event time variability into account and evaluate its impact on our results, we generated, for each day of data, five different downsampled dataset, one per inter-event time value. The selected average inter-event time values are $4,8,12,16$, and 20 minutes, to cover a large range of average communication rates. The corresponding bias distributions are displayed together in Figure 5. We observe that the larger the average bias is, the more spread is the distribution, with a greater probability for high temporal biases, which was expected with Equation 29. This plot allows understanding that even if the speed estimation method is statistically unbiased, the increasing dispersion of the individual biases makes it necessary to have larger samples when working with important average inter-event times compared to small ones. This especially justifies the data expansion led above. Downsampling the expanded trips sample then allows obtaining an extended bias distribution, for which the average bias will be more representative. For each trip, we sample the departure and arrival biases according to the probability density function obtained in Equation 26. We generate a second dataset, referred to as $D S_{1}$, which includes the same trips as in $D S_{0}$ but whose actual travel time information is biased by the sum of the sampled departure and arrival biases to obtain the observed travel time. This dataset records partially biased trips. It will be the subject of our second analyses to assess our ability to correct for travel time bias. In a final downsampling step, we generate a dataset $D S_{2}$ in which the actual arrival time is additionally biased with the sampled arrival bias. $D S_{2}$ records synthetic UADP-extracted trips: individual trips characterized by fully biased temporal features and low-quality path information.

One last step before applying the methodology to any of those two datasets consists of grouping the data by regional paths and 15-minutes periods, and averaging the travel time on the resulting groups.

\subsection{Speed baseline}

We divide the experiment duration into equal periods of 15 minutes. At each time step $t$, the method, applied to one of the datasets describe before, returns a vector $\boldsymbol{V}^{t}$ whose dimension is equal to the number of regions, in this instance 16. A speed reference is needed to validate our method and estimate the impact of the data downscaling and downsampling processes on the reliability of the results. The spatial mean speed $V_{r}^{t}$ in region $r$ over a period $t$ is defined as the ratio of the total traveled distance in region $T T D_{r, t}$ and the total travel time $T T T_{r, t}$ in region $r$ during $t$ :

$$
V_{r, t}=\frac{T T D_{r, t}}{T T T_{r, t}}=\frac{\sum_{i} d_{r, t}^{i}}{\sum_{i} t_{r, t}^{i}}
$$

$T T D_{r, t}$ corresponds to the sum of the individual travel distances in region $r$ during $t$, i.e., $d_{r, t}^{i}$. $T T T_{r, t}$ corresponds to the sum of the individual travel times in region $r$ during $t$, i.e., $t_{r, t}^{i}$. As the GPS data are map matched (see Section 3.3), they include not only temporal and positional data, but also the inferred sequence of road segments traveled, the 
inferred entrance time on each road link, and the distance traveled on each of them. These characteristics give access to precise individual travel times and distances, and therefore allow obtaining a reliable speed baseline to compare speed estimation results from temporally-biased trip data.

To conclude, using simulated data obtained through downscaling and the downsampling of high-frequency GPS data presents the strategic advantage of offering substantial control over the experimental environment while providing easy access to the necessary distance parameters and the ground truth speed data. The next section exposes the results of the approach on datasets $D S_{0}, D S_{1}$ and $D S_{2}$.

\subsection{Trip length estimation}

Finally, the GPS data is used to estimate the trip length estimation matrix. The entire month of February is used to generate this matrix and ensure robust estimation of trip lengths. As the mobility behaviors are characterized by high redundancy, the trip lengths are mostly unvarying from one month to the other. This means that the trip length matrix can be calibrated using data from a period of time that does not necessarily overlap the time span of the study. This assumption was confirmed by comparing trip length matrices computed in February with a similar matrix computed with March's data. Appendix C exposes the result of this comparison. We will explore the results of the method when estimating travel distance with automatic network analysis in later work. In the meantime, one can refer to the comparison of such trip lengths estimation with GPS data in the work of Batista et al. (2021b).

\section{Results}

The speed estimation method that we propose presents the advantages of relying on few mobility features and hence of being easily applicable to UADP data. However, it is essential to evaluate the extent to which the low data quality impacts the accuracy of the speed estimation. To this purpose, we proceed in three steps.

First, we intend to evaluate the impact of working with mobility data of coarser space and time resolution on the results by assessing the errors when working on the $D S_{0}$ dataset. The significant degrading of the GPS data might impact the results. Evaluating this impact is essential to understand the overall potential of the method on temporallybiased trip data.

In a second step, the method is applied to the partially biased dataset $D S_{1}$. First, we estimate the speed dynamics without de-biasing the temporal system, hence with erroneous travel time information, to evaluate to what extent it is necessary to estimate and remove the travel time bias. Second, we solve the de-biased system, and measure the effectiveness of the de-biasing process to obtain satisfactory speed results.

In a third step, we will apply the method to the fully biased dataset $D S_{2}$. We compare the results obtained when correcting only the travel time bias with the results obtained when correcting both the arrival and travel time biases. While the data expansion of our trip sample has no impact on the evaluation when using dataset $D S_{0}$, because it does not change the average travel times, we will see that this step is of importance when dealing with both biased datasets.

\subsection{Method application to trip data with exact travel time}

We start by applying the proposed methodology to dataset $D S_{0}$, to evaluate in a first step the impact of the spatial aggregation and of the speed estimation method.

By using our methodology, we obtain a speed profile in kilometers per hour, per region, and per 15-minutes slots for each day of the evaluation. These speed profiles are compared to the corresponding speed baseline to compute errors. We begin by measuring daily error indicators that characterize the global results of the methodology for the overall regions and periods of the day. We evaluate the mean absolute error (MAE), the root mean absolute error (RMSAE), the mean absolute percentage error (MAPE), and the root mean square absolute percentage error (RMSAPE). Those daily indicators are displayed in Table 2.

It is interesting to observe that the daily errors are substantially similar from one day to another.

To better assess the performance of the methodology, we now focus on one specific day from our day-set, e.g., Day 1 (Monday, February 12, 2018). Comparable results were obtained for the other days. Figure 6 illustrates the speed estimations dynamics obtained for this day. Each of the subplots corresponds to a region of our partitioning of 


\begin{tabular}{lrrrr}
\hline & MAE $(\mathrm{km} / \mathrm{h})$ & RMSAE $(\mathrm{km} / \mathrm{h})$ & MAPE $(\%)$ & RMSAPE $(\%)$ \\
\hline Day 1 & 4.727834 & 6.548142 & 13.111325 & 16.875215 \\
Day 2 & 4.820239 & 6.725782 & 13.169344 & 16.739542 \\
Day 3 & 4.906018 & 7.048133 & 13.486273 & 17.370823 \\
Day 4 & 4.781254 & 6.909102 & 14.909294 & 23.519969 \\
Day 5 & 4.876119 & 6.538737 & 14.013252 & 17.780414 \\
\hline
\end{tabular}

Table 2: Daily speed errors when applying method to $D S_{0}$

Lyon. Time throughout the day is represented on the x-axis in hours while the y-axis is for average traffic speed, in kilometer per hour. The ground truth traffic speed, calculated based on the raw GPS dataset, is represented in blue. The orange line corresponds to the raw speed estimation results after bootstrapping. The green line is the result of the moving average filtering that smooths the speed trends. The first ten plots (from Region 0 to Region 9) correspond to the urban regions, while the last six ones characterize the speed dynamics on ring road.

For most of the inner regions, we observe that both the raw and smoothed speed trends well match our ground truth profiles, both during peak hours and off-peak periods. Regions $0,1,2,5$ and 6 display the most accurate speed estimations. Regions 3, 4 and 7 are those among urban regions where the raw results are the least stable, and for which the smoothing process is the least efficient. The traffic speed in these regions are slightly overestimated.

When it comes to the ring road regions (Region 10 to Region 15), we observe a more significant variability of the raw results, with saw-tooth raw speed estimations. For those regions, we observe that the speed estimates reproduce well the speed trends, generally following the ground-truth speed surges and drops during peak hours and matching the faster speeds in-between. Regions 12, 14 and 15 display the most accurate speed estimations. During peak hours, the raw results display important speed drops, and the increased sensitivity of the filter during assumed peak hours (6 AM to $9 \mathrm{AM}$ and $3 \mathrm{PM}$ to $7 \mathrm{PM}$ ) proves efficient to reproduce these dynamics while smoothing the results. In Regions 12, 14 and 15 the speed estimates reproduce well the speed drops. The speed estimates in Region 10 during the morning drop are also satisfactory. Region 11 is the most concerning at the peak time, as both its morning and afternoon speed trends are overestimated. After investigating this issue, we suppose that the divergence of the southern end of this section of the ring road, on the one hand, towards Region 12, and on the other hand, towards a freeway, with distinct road behaviors, could be the cause of this anomaly. In the future, we aim at looking more thoroughly at the characteristics of this region and explore how its network features might impact our results in this way. Although some smoothed speed trends in other regions miss reproducing the speed drops to their full magnitude (see in particular: Region 13, morning peak or Region 15, afternoon peak), the deviation from the baseline is much lower than the one related to Region 11, and the results remain satisfactory. As the raw speed estimates reach the lower speeds (Region 11, Region 15), modifying the filter during this time window to make it even more sensitive to lower speeds can be a way to reduce this gap and further improve results.

In-between the peak periods, the raw results follow the speed baseline and reproduce its speed dynamics. Regions 10 and 13 display the largest deviations from baseline with a general under-estimation of the speed during this time window. It is interesting to notice that those regions correspond to the opposite directions of the same section of the ring road: section North-East. Region 10 corresponds to the clockwise direction, while Region 13 corresponds to the counterclockwise direction. The reason for this under-evaluation of the speed is that both those regions are strongly connected to the north-western part of the ring road, which was not considered in this study as it mostly corresponds to tunnels. Hence, the process of filtering the scattered tracks related to this north-western section impacted the number of available trips in regions 10 and 13 more than the other ring road regions. In fact, we observe that the frequentation in those regions is, on average, $10 \%$ lower than in the other ring road regions This low frequentation leads to poor representativeness of the average travel time and generates a distance bias between actual and estimated travel distance per region and path. The other ring road regions display satisfactory results during this time window, for which the moving average succeeds in smoothing the raw results and their saw-tooth shape (Regions 11, 12, 14, and 15). However, this filter may be unsuitable if sudden and unexpected speed drops occur outside of peak periods. Despite this limitation, this filter was fast and easy to implement choice. In future work, we will explore other filtering techniques that both allow filtering the small saw-tooth instabilities of the results without neglecting the unexpected 

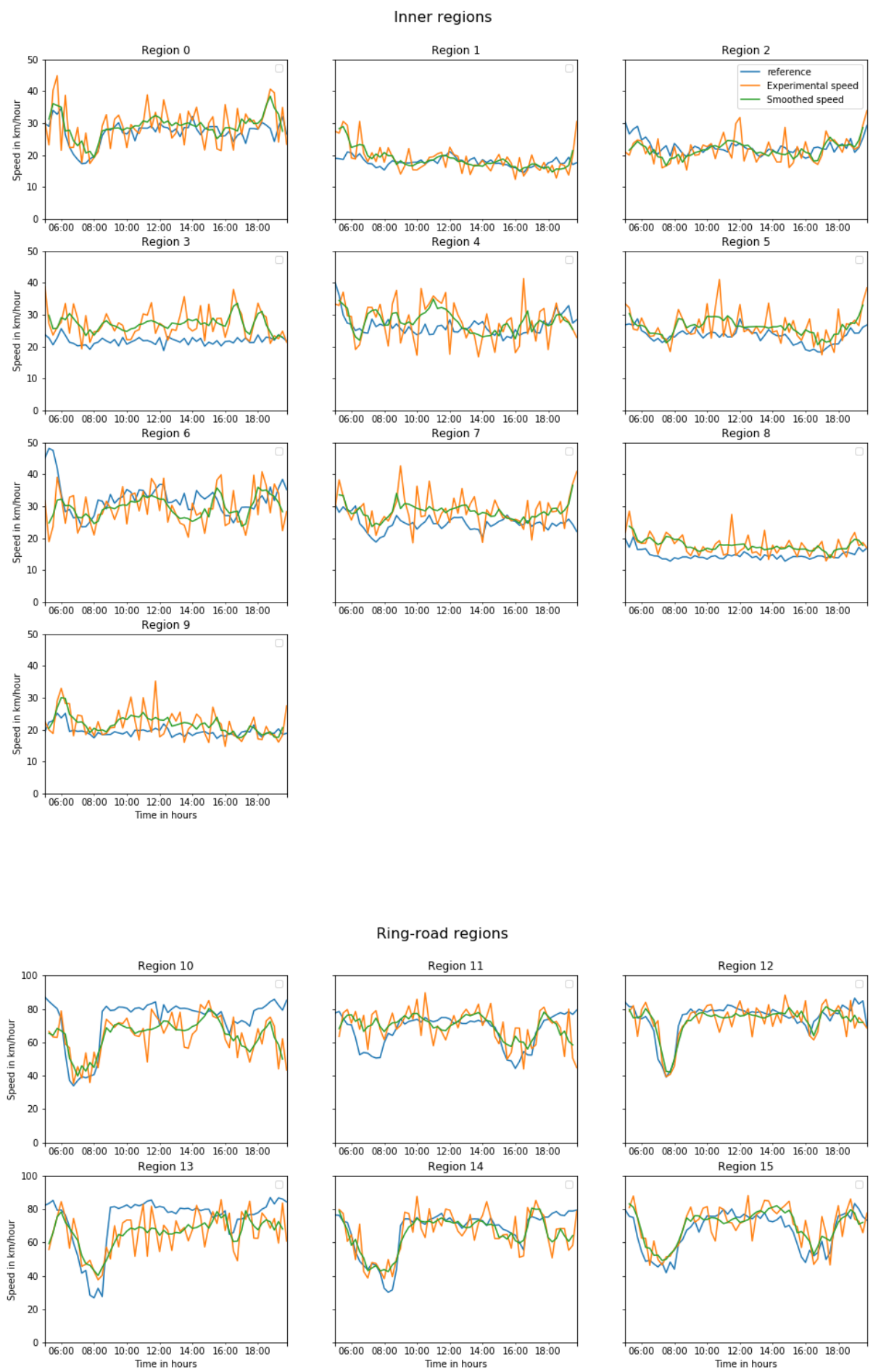

Figure 6: Speed estimation method applied to dataset $D S_{0}$ (trips with exact travel times)) 


\begin{tabular}{lrrrrrr}
\hline & \multicolumn{2}{c}{ MAE $(\mathrm{km} / \mathrm{h})$} & & MAPE $(\%)$ & & \\
& All regions & Inner regions & Ring road & All regions & Inner regions & Ring road \\
\hline Full day & 4.727834 & 3.157841 & 7.344489 & 13.111325 & 14.274299 & 11.173036 \\
\hline Off peak & 4.641103 & 3.090886 & 7.224797 & 12.040770 & 13.794607 & 9.117708 \\
Peak hours & 4.803374 & 3.216156 & 7.448737 & 14.043745 & 14.692095 & 12.963161 \\
\hline Morning peak & 5.354016 & 3.665842 & 8.167639 & 16.491855 & 16.713291 & 16.122797 \\
Afternoon peak & 4.287147 & 2.794576 & 6.774766 & 11.748641 & 12.797223 & 10.001003 \\
\hline
\end{tabular}

Table 3: Speed MAE and MAPE detailed by region and time window for Day 1

\begin{tabular}{lrrrr}
\hline & MAE $(\mathrm{km} / \mathrm{h})$ & RMSAE $(\mathrm{km} / \mathrm{h})$ & MAPE $(\%)$ & RMSAPE $(\%)$ \\
\hline Day 1 & 3.780724 & 5.308405 & 10.711582 & 14.277904 \\
Day 2 & 3.698802 & 5.013979 & 10.599366 & 13.624562 \\
Day 3 & 3.706125 & 5.343630 & 10.516593 & 13.799747 \\
Day 4 & 4.061264 & 5.876281 & 12.163860 & 18.042898 \\
Day 5 & 4.078924 & 5.792345 & 11.312538 & 14.590598 \\
\hline
\end{tabular}

Table 4: Daily speed errors when applying method to $D S_{0}$, using the actual trip lengths instead of static trip length estimates

speed drop that may occur at any time of the day.

In Table 3, we detail the MAE and MAPE errors by period and region type for Day 1. Those errors are computed from the smoothed results. We observe that while the daily error is bigger in absolute value in the ring road regions than in urban regions, the absolute percentage error is smaller for the ring road. Generally speaking, the percentage errors are higher in peak hours than during the off-peak period. However, we also notice that its value is smaller for ring road regions than in inner ones, showing that the method is quite efficient in reproducing the fast-changing speeds of this particular kind of region.

Those results are interesting as they give a first insight into the potential of the method. Despite significantly lowering the information carried by individual trips (from GPS tracks to regional paths, and from exact arrival time to arrival period), the method reproduces the speed trends with limited errors. From the perspective of estimating traffic speed from temporally sparse data, this is a promising step.

However, we can identify several potential improvements. We already mentioned the improvements concerning the smoothing filter. The specific characteristics of Region 11 are also under investigation to understand how they impact the results. More generally, we can only stress the importance of the sample size. In fact, the number of individuals traveling along a regional path at each step must be large enough for the exogenously computed mean travel distance to represent the sample and for the sample's average travel time to represent the instantaneous dynamics along the path. When working with massive data, the amount of data available will ensure this representativeness and compensate for the low data information level. However, working with GPS data present the drawback of having to deal with a limited amount of tracks, and therefore, even more, a limited amount of tracks by regional path and period. This likely results in distance biases between the estimates and the actual average traveled distance, destabilizing the results. Hence, this case study can be considered a worst-case scenario in which the method requires us to work with limited access to trip information.

We explored the same speed estimation process from dataset $D S_{0}$ when replacing the static trip length estimates by the actual travel distances to validate those considerations. The speed trends for Day 1 are displayed in Figure 7 , while the corresponding daily errors can be found in Table 4. The important improvement we observe, especially for Regions 10 and 13, confirms that a finer representativity of the trip length estimates should allow for more accurate results, thus limiting the gaps to the baseline. For this reason, and despite the limitations we mentioned, the method is very promising for an application to a way larger dataset. 
Inner regions
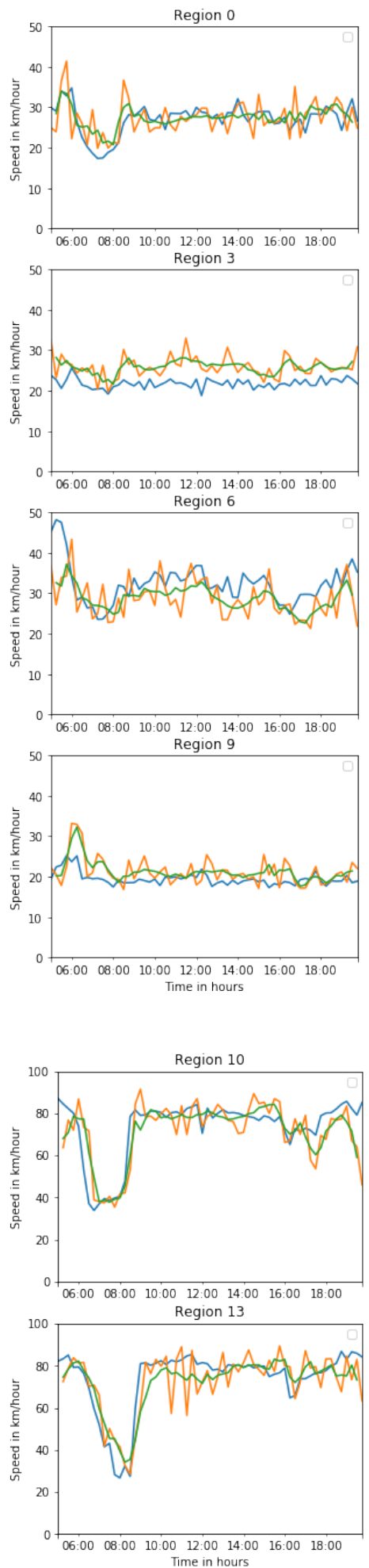

Region

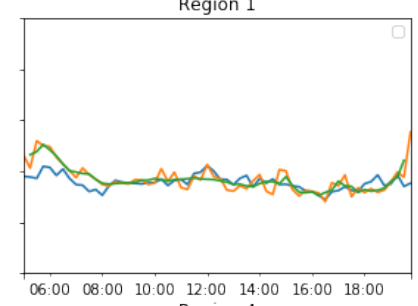

Region 4

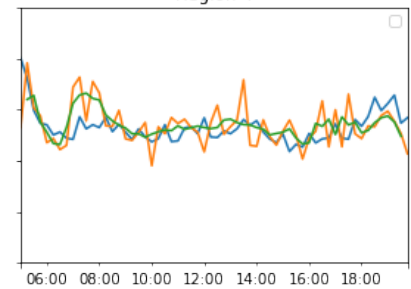

Region 7

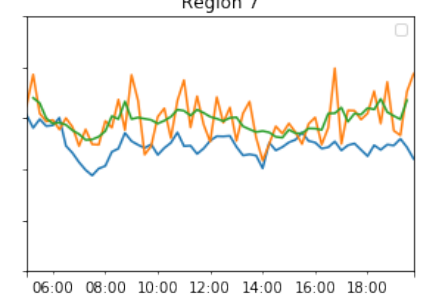

$\begin{array}{lllllll}06: 00 & 08: 00 & 10: 00 & 12: 00 & 14: 00 & 16: 00 & 18: 00\end{array}$

Ring-road regions

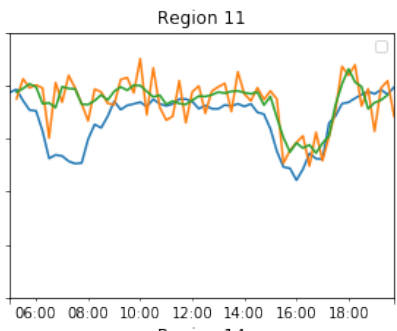

Region 14

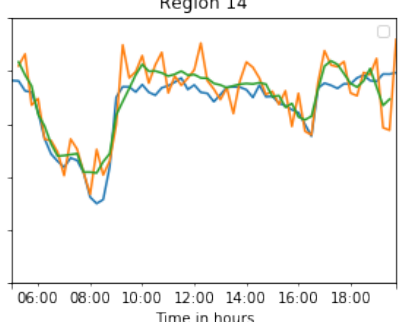

Region 2

- reference

- Experimental speed

— Smoothed speed

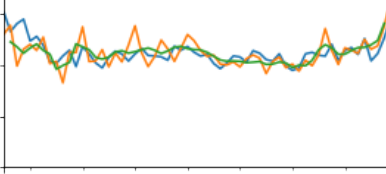

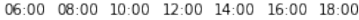
Region 5
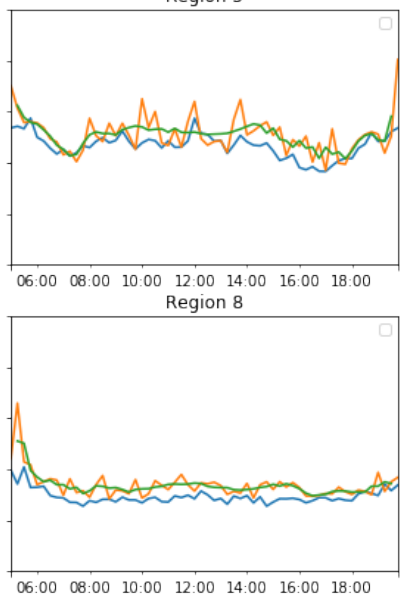

Figure 7: Speed estimation method applied to dataset $D S_{0}$ (trips with exact travel times), using the dynamic trip lengths 


\begin{tabular}{lrrrr}
\hline Avg IET & MAE $(\mathrm{km} / \mathrm{h})$ & RMSAE $(\mathrm{km} / \mathrm{h})$ & MAPE $(\%)$ & RMSAPE $(\%)$ \\
\hline 4 & 7.894771 & 18.576767 & 10.773218 & 21.648403 \\
8 & 12.970168 & 31.347989 & 16.233049 & 33.484246 \\
12 & 16.918677 & 41.133133 & 20.600466 & 42.693440 \\
16 & 19.920772 & 48.522253 & 24.008746 & 49.748655 \\
20 & 22.204453 & 54.189049 & 26.608480 & 55.214964 \\
\hline
\end{tabular}

Table 5: Speed errors on average over the week for each mean inter-event time selected as downsampling parameter
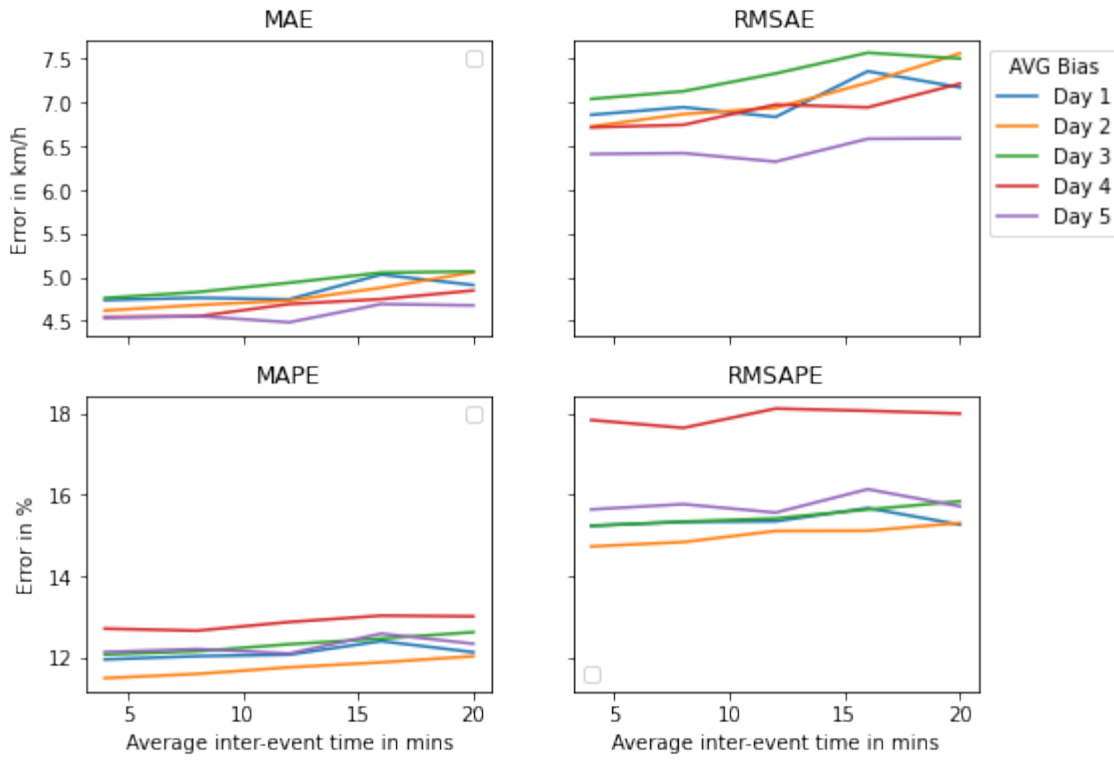

Figure 8: Evolution of daily errors with increase of average bias

\subsection{Method application to trip data with biased travel time}

In this section, we evaluate our method on the trip dataset $D S_{1}$ made of trips with biased travel times. First, we compute the zonal traffic speeds without applying the temporal bias removal. This allows evaluating the impact of the temporal imprecisions on the results. The variations of average errors over the week with average inter-event time are gathered in Table 5. We observe a significant increase in the errors, compared with Table 2. This shows how a bad estimation of travel times deteriorates the results' quality, even with a short average inter-event time and a limited travel time increase. It justifies the need for de-biasing in average the travel times. The following results are computed applying this de-biasing process.

We display in Figure 8 the evolution for each day of the different daily error indicators as a function of the average inter-event time. We observe that the error indicators are quite stable and rise slowly with the average inter-event time. On the contrary, when not expanding the data, the error increases quickly due to the increased dispersion of the bias distribution with the average inter-event time. This shows how important the sample size is and proves the capacity of a large dataset to compensate for the individual biases and imprecision and keep the bias removal process useful despite a large bias dispersion.

Figure 9 displays the smoothed results of the speed estimation for the five different average inter-event time values. The results for each value of average inter-event time almost fall into the same line, which confirms the aforementioned results. We observe that we are able, once again, to reproduce the traffic trends and dynamics.

In urban regions, the results are satisfactory in Regions 2, 5, 6, 8 and 9. In Regions 0, 1, 3, 4 and 7, the results are less consistent with the speed baseline. While the Region 3, 4 and 7 were already identified in the previous section as 

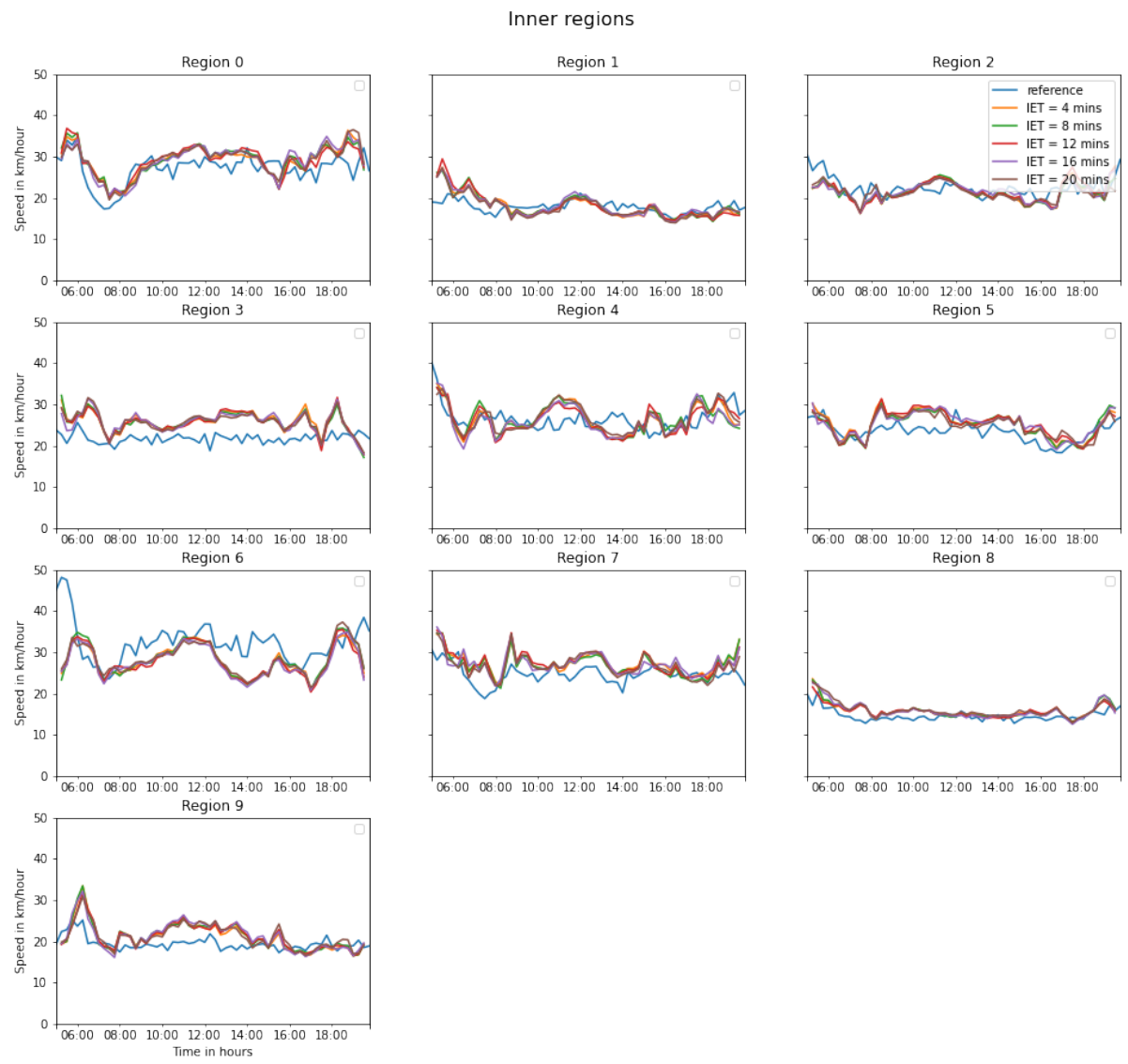

$06: 00$ 08:00 10:00 12:00 14:00 16:00 18:00
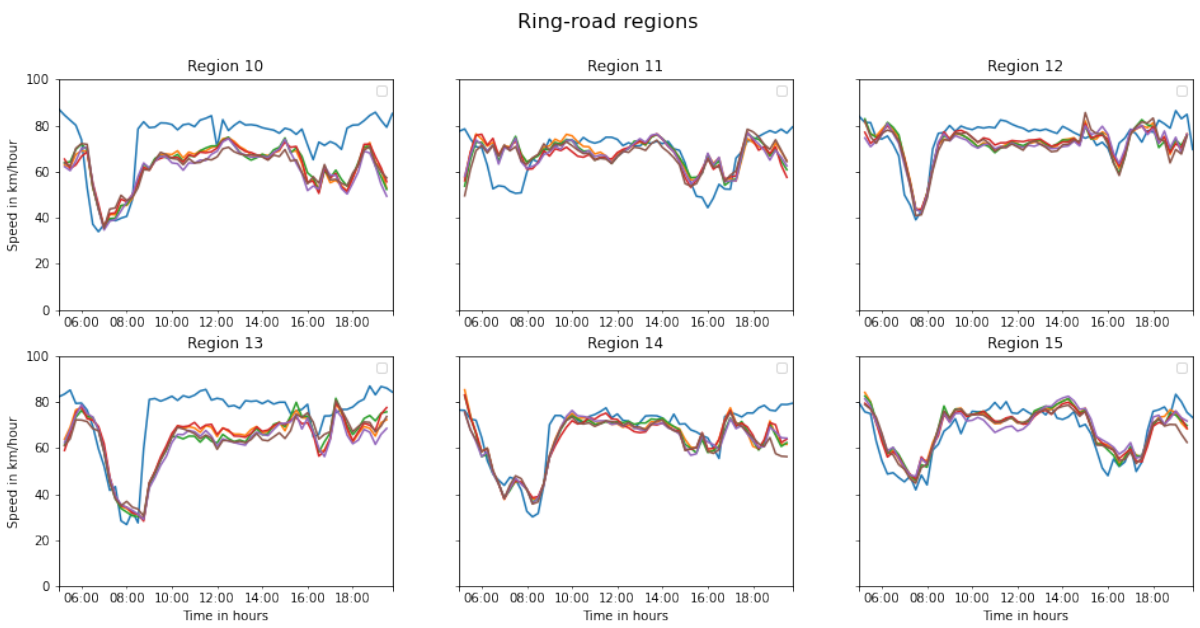

Figure 9: Speed estimation method applied to dataset $D S_{1}$ (downsampled trips) after average bias removal. 


\begin{tabular}{lrrrr}
\hline & MAE $(\mathrm{km} / \mathrm{h})$ & RMSAE $(\mathrm{km} / \mathrm{h})$ & MAPE $(\%)$ & RMSAPE $(\%)$ \\
\hline Day 1 & 4.911907 & 7.174379 & 12.124498 & 15.264945 \\
Day 2 & 5.057217 & 7.564531 & 12.024699 & 15.306312 \\
Day 3 & 5.067316 & 7.505059 & 12.617445 & 15.839542 \\
Day 4 & 4.848885 & 7.216612 & 13.006559 & 18.000039 \\
Day 5 & 4.678821 & 6.591109 & 12.331655 & 15.714796 \\
\hline
\end{tabular}

Table 6: Daily speed errors when applying our method to $D S_{1}$ in the worst bias scenario (IET $=20$ mins)

\begin{tabular}{|c|c|c|c|c|c|c|}
\hline & \multicolumn{3}{|l|}{$\operatorname{MAE}(\mathrm{km} / \mathrm{h})$} & \multicolumn{3}{|l|}{ MAPE (\%) } \\
\hline & All regions & Inner regions & Ring road & All regions & Inner regions & Ring road \\
\hline Full day & 4.911907 & 2.794057 & 8.441657 & 12.124498 & 12.113224 & 12.143288 \\
\hline Off peak & 5.181909 & 2.790098 & 9.168261 & 11.769294 & 11.884125 & 11.577910 \\
\hline Peak hours & 4.676744 & 2.797506 & 7.808808 & 12.433869 & 12.312762 & 12.635715 \\
\hline Morning peak & 4.961260 & 3.236535 & 7.835803 & 14.194493 & 14.174446 & 14.227904 \\
\hline Afternoon peak & 4.410010 & 2.385915 & 7.783501 & 10.783285 & 10.567434 & 11.143037 \\
\hline
\end{tabular}

Table 7: Speed MAE and MAPE detailed by region and time window for Day 1 in the worst bias scenario (IET $=20 \mathrm{mins}$ )

displaying less satisfactory results, the increases of the errors for the Regions 0 and 1 can be related to the introduction of the bias.

In the ring road regions, most estimated speed trends follow the speed baseline. The speed trends are particularly similar to the baseline in Regions 12, 14 and 15, although some speed drops are not reproduced with their full magnitude (Region 15, especially), but it was mostly already the case when working with unbiased data. Unsurprisingly, the results in Regions 10 and 13 remain underestimated in-between the peak periods, similarly to the case with unbiased data, but the speed drops are clearly observed. The estimation errors that we had already observed for Region 11 during peak hours in the case of unbiased data are increased when using biased data.

Finally, we further analyze the worst-case scenario results with an average inter-event time of 20 minutes. Table 6 displays the average errors observed for each day in this case, while Table 7 details the precise errors by region type and time window. Compared to the previous section results, we notice a general increase in the errors, although limited. The errors remain under a 20\% limit when considering the daily RMSAPE, which is acceptable even though there is room for improvement here.

Overall, taking into account the errors previously introduced by upscaling of the GPS tracks to the regional path, working with biased trips seems to have a limited negative impact on the result. Despite the low quality of the trip information at this stage, the results are very encouraging. Therefore, the room for improvement includes the reduction of errors at each stage of the process. This ranges from the representativeness of trip length and time estimates, to the filtering process, to a more refined understanding of the impact of internal speed dynamics in the results.

\subsection{Method application to trip data with both biased arrival and travel time}

In the preceding section, we have analyzed our results when using trips with a biased travel time information. However, UADP data not only display biases in the travel time, but on the arrival time as well. In this section, we therefore consider this additional bias on the trips by exploiting the $D S_{2}$ dataset, and explore the impact of the methods we propose on such results.

First, we compute the results of our method on dataset $D S_{2}$ when handling the travel time bias only. The results are displayed in Table 8. Compared to Table 6 for instance, which represents the average errors we obtained for each day in the worst case scenario, those results display a new significant increase of the errors. Since the arrival times are de-skewed, this increasing of the errors is related to the arrival time bias only, which results in mixing together users traveling at different periods and in erroneous travel time estimations. Without surprise, we can observe that the 


\begin{tabular}{lrrrr}
\hline Avg IET & MAE $(\mathrm{km} / \mathrm{h})$ & RMSAE $(\mathrm{km} / \mathrm{h})$ & MAPE $(\%)$ & RMSAPE $(\%)$ \\
\hline 4 & 6.402842 & 14.587325 & 9.344040 & 17.957539 \\
8 & 10.950956 & 24.768931 & 14.947251 & 28.044663 \\
12 & 14.044534 & 32.729041 & 18.393624 & 35.744618 \\
16 & 16.329243 & 38.268281 & 21.118529 & 41.143766 \\
20 & 17.901683 & 42.022657 & 23.004366 & 44.958863 \\
\hline
\end{tabular}

Table 8: Speed errors on average over the week for each mean inter-event time selected as downsampling parameter
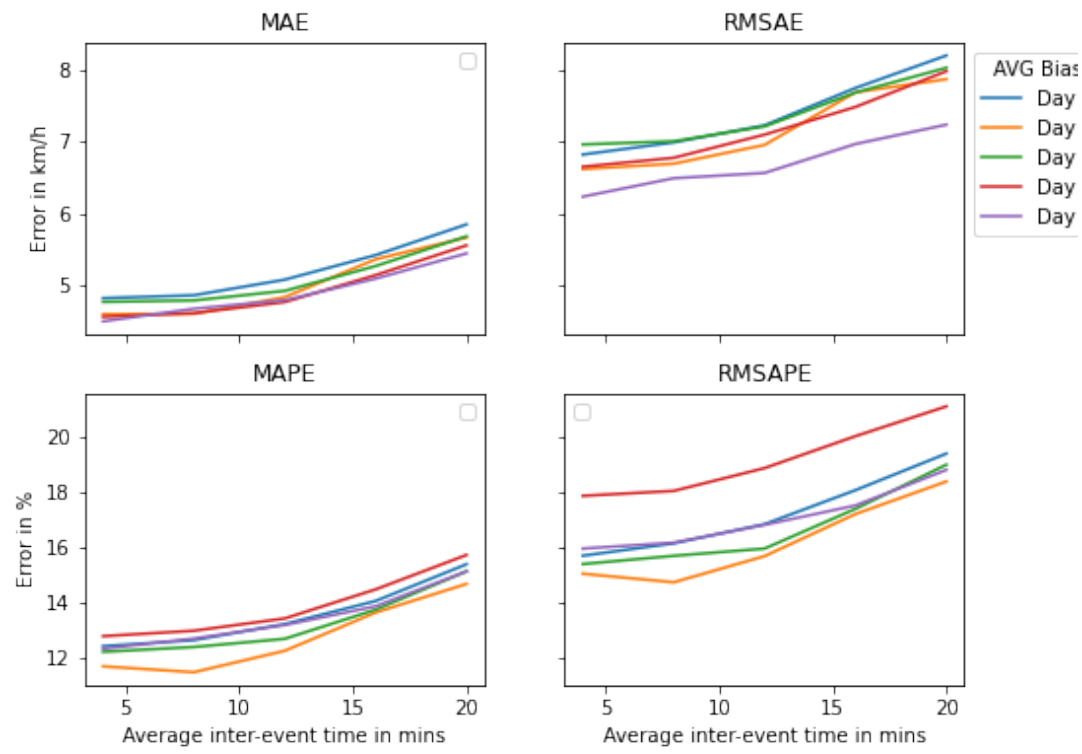

Figure 10: Evolution of daily errors with increase of average bias

larger the average inter-event time is, the larger the errors are, because trips are shifted further away from their actual travel time period.

Therefore, handling this arrival time uncertainty seems necessary, as it was previously done with the travel time. This is what we address in the second part of this section. Figure 10 displays the results obtained once we shift back each trip's arrival time by $\mu_{X} / 2$, remove users with bias larger than twice the average bias and filter regional paths that represent less than 30 individuals.

Although we still observe a sensibility to the average inter-event time (and average bias), the results are contained within much lower bounds than the ones observed in Table 8, showing the filters' efficiency in limiting the arrival time bias impact on the results. However, compared to Figure 5, we observe a larger increasing of the error with the average bias, which can be explained by the fact that the larger the average bias is, the larger the variance, resulting in an increased data shuffling.

In Figure 11, we display the speed estimation results obtained for each inter-event time value on Day 1. These plot display results that can reasonably be compared to the ones exposed in 9 . Table 9 precise the daily results in the worst case scenario, while Table 10 precise the errors by region and time period. Overall, the increasing of the errors compared to Tables 6 and 7 is limited, which confirms the viability of our method for estimating regional traffic speeds despite low-quality path information and fully biased temporal features. In particular, these latest analyses demonstrate the utility of implementing filters at the individual and equation levels to compensate for the temporal biases of the data. This suggests that these filters will have great potential when it comes to handling large amounts 

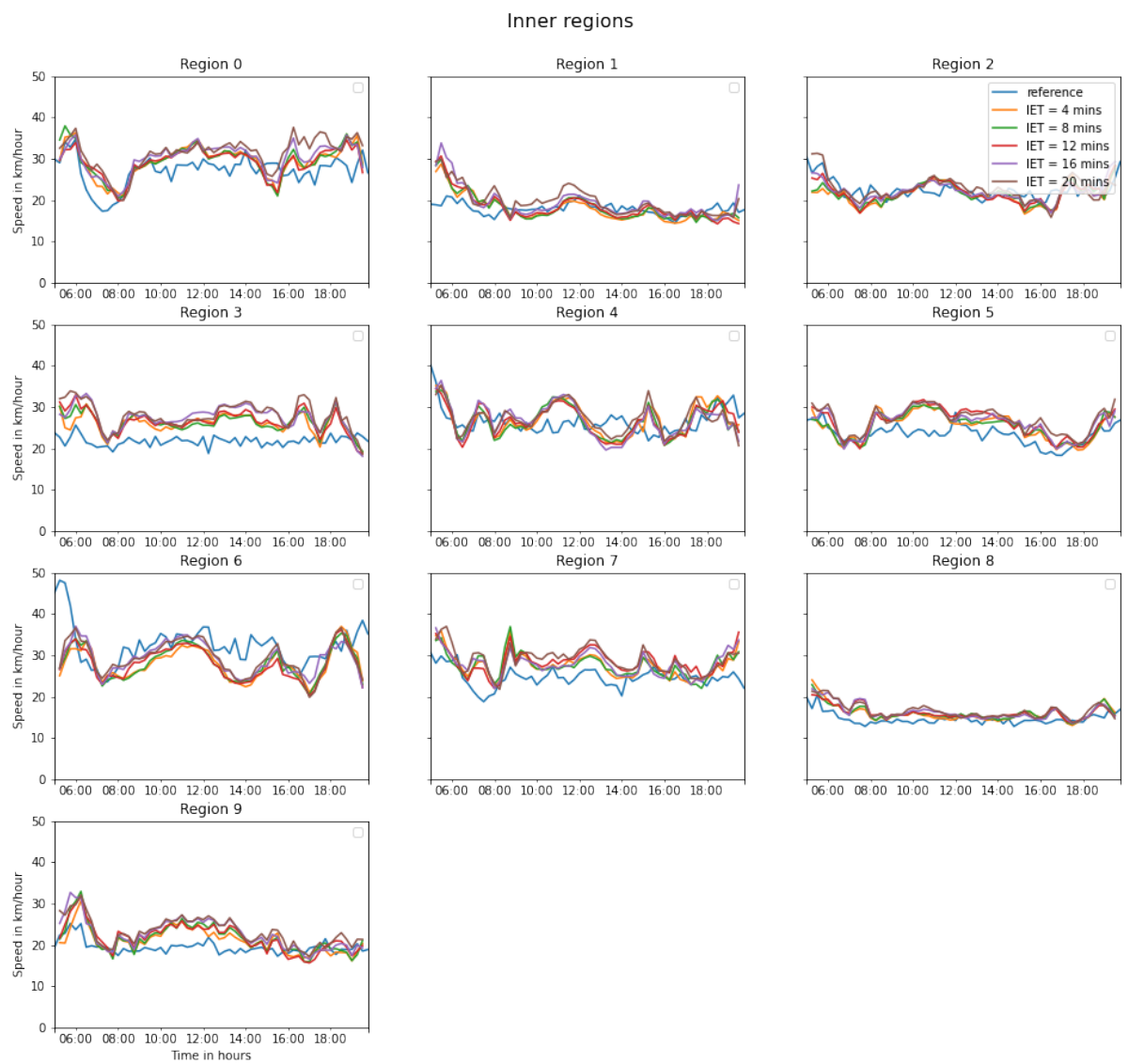

$06: 00 \quad 08: 00 \quad 10: 00 \quad 12: 00 \quad 14: 00 \quad 16: 00 \quad 18: 00$
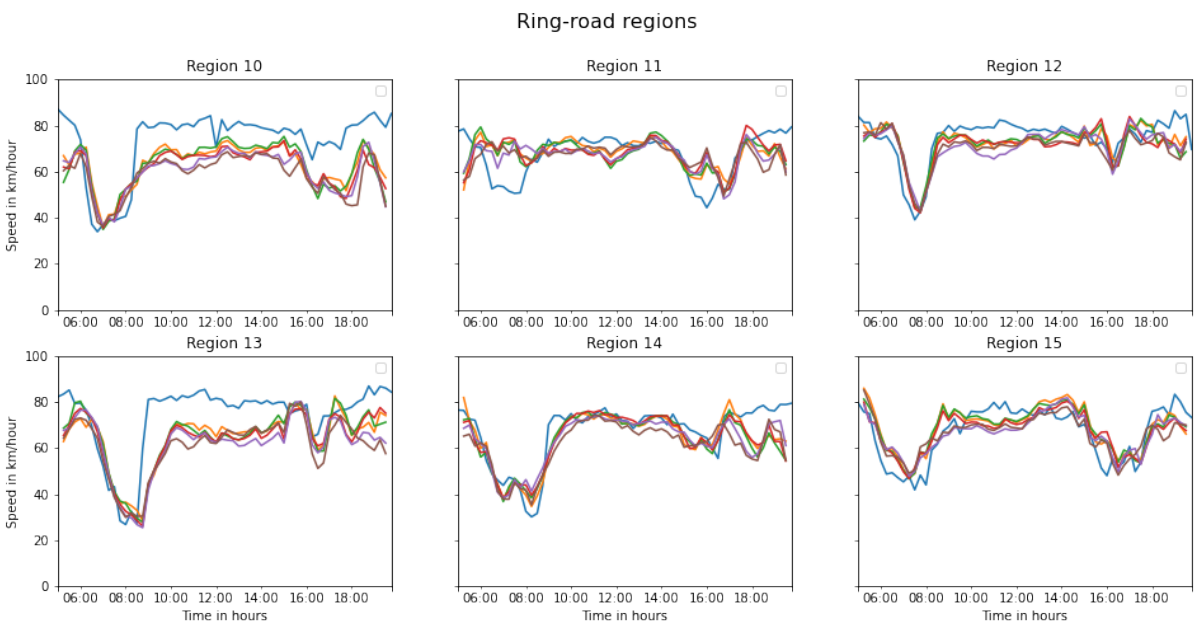

Figure 11: Speed estimation method applied to dataset $D S_{2}$ (fully biased trips) after average bias removal and arrival time correction. 


\begin{tabular}{lrrrr}
\hline & MAE $(\mathrm{km} / \mathrm{h})$ & RMSAE $(\mathrm{km} / \mathrm{h})$ & MAPE $(\%)$ & RMSAPE $(\%)$ \\
\hline Day 1 & 5.843820 & 8.214792 & 15.377055 & 19.398781 \\
Day 2 & 5.659251 & 7.883027 & 14.660121 & 18.385150 \\
Day 3 & 5.676346 & 8.042682 & 15.119238 & 18.995601 \\
Day 4 & 5.549316 & 7.994296 & 15.714570 & 21.116936 \\
Day 5 & 5.436428 & 7.243572 & 15.116918 & 18.807797 \\
\hline
\end{tabular}

Table 9: Daily speed errors when applying method to $D S_{2}$, when in the worst bias scenario (IET = 20 mins)

\begin{tabular}{lrrrrrr}
\hline & \multicolumn{2}{l}{ MAE $(\mathrm{km} / \mathrm{h})$} & & MAPE $(\%)$ & & \\
& All regions & Inner regions & Ring road & All regions & Inner regions & Ring road \\
\hline Full day & 5.843820 & 3.720738 & 9.382290 & 15.377055 & 16.573524 & 13.382939 \\
\hline Off peak & 5.999818 & 3.771147 & 9.714271 & 14.918967 & 16.531425 & 12.231538 \\
Peak hours & 5.707951 & 3.676834 & 9.093144 & 15.776034 & 16.610191 & 14.385773 \\
\hline Morning peak & 5.621608 & 4.077899 & 8.194454 & 17.050427 & 18.541036 & 14.566079 \\
Afternoon peak & 5.788897 & 3.300836 & 9.935666 & 14.581290 & 14.800023 & 14.216735 \\
\hline
\end{tabular}

Table 10: Speed MAE and MAPE detailed by region and time window for Day 1, when in the worst bias scenario (IET = 20mins)

of data, which we are eager to verify.

\section{Conclusion and discussion}

This paper has proposed a new methodology for estimating the dynamics of regional traffic speeds from useractivity-dependent positioning data. The trips extracted from these data present the challenging issue of being temporally biased, making the individual traffic speed difficult to estimate. To address this issue, the method we propose first relies on the definition of a proper data resolution scale, both on the temporal and spatial dimension, which is used to group and aggregate the user-activity-dependent positioning data. It especially requires the partitioning of the studied area in sub-regions characterized by homogeneous traffic. Such partitioning allows defining basic trip features, such as regional paths, which allow the identification and aggregation of similar trips. This aggregation allows a systematic, exhaustive, and robust estimation of average travel times throughout the network and at each time step through the fusion and de-skewing of individual travel times. Finally, provided that estimates of the regional trip lengths have been performed beforehand, the travel time estimations are jointly analyzed to deduce the underlying regional traffic speeds. This structure of the method is particularly fitted to any massive but temporally sparse data input, as it requires very little temporal or itinerary information at the individual level and considers the inherent temporal bias that characterizes trips extracted from those data.

Applying this approach to downsampled GPS data offers a controlled environment to evaluate the different degrading steps of our approach. First, despite reducing the available GPS trips to minimal temporal and path information, the method could reproduce the speed trends throughout the day, especially the fast-changing dynamics observed in the ring road regions. The moving average smoothing filter that we implemented in this article was proved to be efficient to smooth the period-to-period instabilities of the results. More elaborated filters can replace this one in future work. Despite these satisfactory results, two regions, in particular, displayed underestimated speeds, which we related both to their representation level and inner dynamics. After the introduction of individual temporal biases on the travel time, we repeated the experiment. Different bias models were explored by making the average inter-event bias vary between 4 (best case) to 20 (worst case) minutes. We showed that, provided that the amount of data was sufficient for the mean bias to be representative of the individual sample, the system could be de-biased and return satisfactory results, although we noted that the error slightly increased. In the last step, the method was applied on trips for which both the observed travel and arrival times were biased. At each step, we have identified methodological options that could help to reduce these errors. Working with a large amount of data was identified as an essential requirement 
of the method. Indeed, it ensures both good reliability of the systems' equations and a correct de-biasing process, especially when working with datasets characterized by long average inter-event time. The sample size was an issue in our case study, in which we had to deal with both low data availability (related to the GPS data source) and the poor quality we imposed on the data to replicate the characteristics of the data UADP. This problem was circumvented by artificially increasing the size of the trip data set by duplicating each displacement 100 times. When working with massive UADP datasets, this problem should no longer arise because the amount of data per regional path will be much more significant, allowing for greater representativeness of displacement lengths and adequate management of bias dispersion. To further investigate the first aspect, we also showed how more accurate dynamic trip length estimates could reduce errors. It is a promising research direction as several studies in the literature have shown that regional trip lengths are relatively stable from day to day but can experience variations within days related to the congestion spreading (Batista et al., 2021a; Paipuri et al., 2021).

In future works, we first would like to explore the sensibility of our method to the different parameters such as the size of the regions or the period duration. We also look forward to testing the robustness and the portability of our method in other geographical contexts. Most importantly, we plan to apply the methodology to user-activitydependent positioning data, tackling the challenges that GPS data have allowed us to leave aside so far. Despite the promising results of our method, the gaps to fill for reaching this objective are still significant. A significant effort will have to be put into the data preprocessing. This step has not been considered in this work because of the high adaptation to the input data it requires. Although the requirements concerning the trip database are limited, this preprocessing step must not be undermined. Besides data cleaning and smoothing, it will have to especially handle mode detection and reconstruction of regional paths from sparse positioning data. The former question is an essential aspect of the preprocessing phase because it distinguishes vehicle movements from other users who do not contribute to road traffic. However, it also corresponds to a significant scientific challenge considering the sparse temporal resolution of UADP data. The review produced by Huang et al. (2019) provides insight on this issue. Methods such as trip reconstruction, individuals' habits completion from (Chen et al., 2019), or matching with prevalent itineraries at the population scale may be helpful (Batista et al., 2021b) to address the latter question on regional path detection.

Above all, we would like to address the critical assumptions concerning the travel time bias we made in this study. The average value was assumed to be known and considered static in time and invariant to space. While the temporal characterization of inter-event times in UADP data has been explored in several works, the specific question of the bias existing between observed and actual travel times when working with trips derived from these data has, to the best of our knowledge, never been explored by the literature. Therefore, the assumed characteristics of the temporal bias are difficult to confirm or invalidate. The leads for the estimation of such a bias are also limited. Although our method only requires an estimate of the average bias and not a full characterization of its distribution, the lack of literature on the subject limits the immediate application of this paper. In this paper, we have proposed a simplistic model relating this bias with the inter-event time distribution. Although the objective of this model was mainly to provide a methodological context for the sub-sampling of data, we believe that the characterization of this bias does indeed require relating it to the inter-event time distribution. We would like to investigate this question further.

\section{Acknowledgements}

This study is part of a larger research and development project, the Green City Big Data project, carried out by the Citepa, Paris, France.

\section{Authors contributions}

M. Seppecher contributed to the conceptualization, methodology, data curation, results analysis, validation, and writing of the original draft of the paper. L. Leclercq contributed to the conceptualization, methodology, results analysis, and review \& editing of the paper. A. Furno contributed to the methodology, results analysis, and review \& editing of the paper. D. Lejri contributed to the methodology, results analysis, and review \& editing of the paper. T. Vieira da Rocha contributed to the funding acquisition, project administration and review of the paper. All the authors have approved the final version of this paper submitted to publication. 


\title{
" Appendices
}

\author{
A. Table of notations
}

Table A.1 summarizes the notation used in this paper.

Table A.1: Nomenclature used in this paper.

General notations:
$P$
$r$
$t$

Generic regional path

Generic region

Generic time period

Individual trip characteristics:

$i \quad$ Generic individual trip

$t_{0}^{i} \quad$ Actual arrival time of trip $i$

$t_{0, o b s}^{i} \quad$ Observed arrival time of trip $i$

$t^{i}$

Actual arrival period of trip $i$

$t_{\text {obs }}^{i}$

$T_{(P)}^{i}$

$T_{(P), o b s}^{i}$

$\varepsilon_{d}^{i}$

$\varepsilon_{a}^{i}$

$\varepsilon^{i}$

$V_{r}^{i}$

$L_{P, r}^{i}$

Observed arrival period of trip $i$

Actual travel time of trip $i$ (along $P$ )

Observed travel time of trip $i$ (along $P$ )

Temporal bias of trip $i$ existing between observed departure time and actual one

Temporal bias of trip $i$ existing between actual arrival time and observed one

Travel time bias on trip $i$

Average speed of $i$ in region $r$

Distance traveled in region $r$ of $P$ by $i$

Travel time estimation:

$I_{P}^{t}$

$n_{t, P}$

$\bar{T}_{P}^{t}$

$\bar{T}_{P, o b s}^{t}$

$\bar{T}_{P, o b s}^{t}$

$T_{P r}^{i}$

$\bar{\varepsilon}_{P}^{t r}$

Overlapping trips along $P$ reaching destination at $t$

Number of trips in $I_{P}^{t}$

Average actual travel time of trips in $I_{P}^{t}$

Average observed travel time of trips in $I_{P}^{t}$

Average travel time bias of trips in $I_{P}^{t}$

Actual travel time of trip $i$ in region $r$

Average bias of trips in $I_{P}^{t}$

Speed estimation:

$V_{r}^{t}$

$\bar{L}_{P, r}^{t}$

Mean spatial speed in region $r$

Average distance traveled in $r$ along $P$ during period $t$

$\hat{L}_{P, r}$

$x_{r}^{t}$

Regional trip length estimate in region $r$ along $P$

Reciprocal of $V_{r}^{t}$

$S^{t}$

Equation system at period $t$

$\hat{L}$

$\hat{L}^{\mid t}$

$T_{o b s}^{t}$

Trip length matrix estimate

Sub-matrix of $\hat{\boldsymbol{L}}$ made of the regional paths observed at period $P$

average observed travel time vector

Solution vector of $S^{t}$ doing a least square regression

Continued on next page 


\section{B. Bias characterization}

We detail here the calculation leading to the results in Section 3.1. In that section, we defined :

$$
\begin{aligned}
Z & \sim \operatorname{Exp}(\lambda) \\
Y \mid Z & \sim U(0, z)
\end{aligned}
$$

Marginalizing over $Z$, the probability density function of $Y$ can be expressed as:

$$
\begin{aligned}
f_{Y}(y) & =\int_{0}^{+\infty} f_{Y \mid Z}(y \mid z) \cdot f_{Z}(z) d z \\
& =\int_{y}^{+\infty} \frac{1}{z} \cdot \lambda e^{-\lambda z} d z \\
& =\lambda \int_{0}^{+\infty} \frac{e^{-\lambda(y+z)}}{y+z} d z
\end{aligned}
$$

The expected value of $Y$ is then calculated as follows:

$$
\begin{aligned}
E(Y) & =\int_{0}^{+\infty} E(Y \mid Z=z) \cdot f_{Z}(z) d z \\
& =\int_{0}^{+\infty} \frac{z}{2} \cdot f_{Z}(z) d z \\
& =\frac{1}{2} \int_{0}^{+\infty} z \cdot f_{Z}(z) d z \\
& =\frac{1}{2} E(Z) \\
& =\frac{1}{2 \lambda}
\end{aligned}
$$

While the variance of $Y$ is given by:

$$
V(Y)=E\left(Y^{2}\right)-E(Y)^{2}
$$

Yet: 


$$
\begin{aligned}
E\left(Y^{2}\right) & =\int_{0}^{+\infty} E\left(Y^{2} \mid Z=z\right) \cdot f_{Z}(z) d z \\
& =\int_{0}^{+\infty} \frac{z^{2}}{3} \cdot f_{Z}(z) d z \\
& =\frac{1}{3} E\left(Z^{2}\right)=\frac{1}{3}\left(V(Z)+E(Z)^{2}\right) \\
& =\frac{1}{3}\left(\frac{1}{\lambda^{2}}+\frac{1}{\lambda^{2}}\right) \\
& =\frac{2}{3 \lambda^{2}}
\end{aligned}
$$

Thus:

$$
\begin{aligned}
V(Y) & =E\left(Y^{2}\right)-E(Y)^{2} \\
& =\frac{2}{3 \lambda^{2}}-\frac{1}{4 \lambda^{2}} \\
& =\frac{5}{12} \frac{1}{\lambda^{2}}
\end{aligned}
$$

\section{Trip Length Matrix Variation with time}

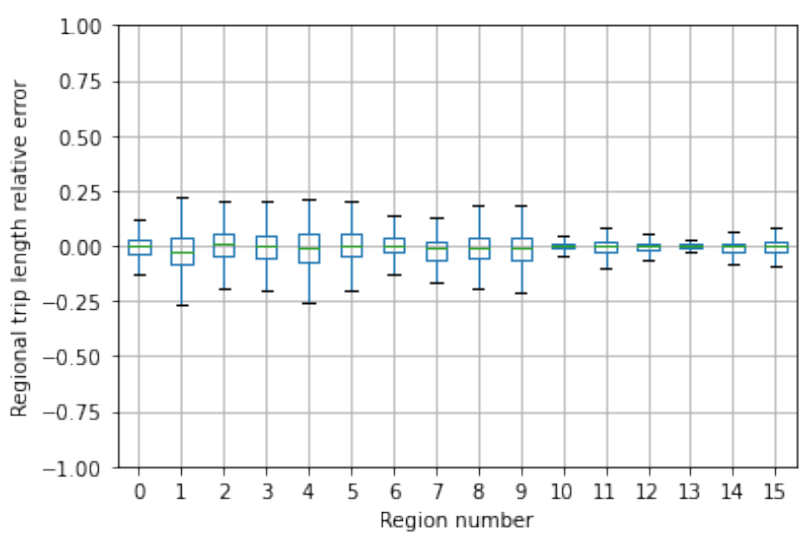

Figure C.1: Box plot of the relative errors of the regional trip lengths by region.

In Figure C.1, we display the boxplot describing for each region the distribution of the relative errors between regional trip lengths computed on February's data and March's data. We observe larger errors in urban regions (Regions 0 to 9), while the ring road regions display lower ones. This observation is related to the fact that the trip lengths distances on the ring road are very constrained by the ring road linear structure. On the contrary, a given regional path has a more extensive range of regional trip lengths in the city center, explaining the larger errors. However, the errors are still bounded in the urban regions, which confirms a regularity of regional average trip lengths overtime. This observation supports our framework, as it guarantees that average trip lengths estimated from another period of time, possibly from an independent dataset, will still provide a reliable database for the speed estimation process. 


\section{References}

Alexander, L., Jiang, S., Murga, M., González, M.C., 2015. Origin-destination trips by purpose and time of day inferred from mobile phone data. Transportation Research Part C: Emerging Technologies 58, 240 - 250. URL: http://www. sciencedirect.com/science/article/pii/ S0968090X1500073X, doi:https://doi.org/10.1016/j.trc.2015.02.018. big Data in Transportation and Traffic Engineering.

Algizawy, E., Ogawa, T., El-Mahdy, A., 2017. Real-time large-scale map matching using mobile phone data. ACM Trans. Knowl. Discov. Data 11. URL: https://doi .org/10.1145/3046945, doi:10.1145/3046945.

Asgari, F., Gauthier, V., Becker, M., 2013. A survey on human mobility and its applications. arXiv:1307.0814.

Bachir, D., Gauthier, V., El Yacoubi, M., Khodabandelou, G., 2017. Using mobile phone data analysis for the estimation of daily urban dynamics, in: ITSC 2017 : 20th International Conference on Intelligent Transportation Systems, IEEE Computer Society, Yokohama, Japan. pp. 626 632. URL: https://hal .archives-ouvertes.fr/hal-01745767, doi:10.1109/ITSC. 2017.8317956.

Bar-Gera, H., 2007. Evaluation of a cellular phone-based system for measurements of traffic speeds and travel times: A case study from israel. Transportation Research Part C: Emerging Technologies 15, 380-391. URL: http://dx.doi.org/10.1016/j.trc.2007.06.003, doi:10. 1016/j.trc.2007.06.003.

Barabási, A.L., 2005. The origin of bursts and heavy tails in human dynamics. Nature 435, 207-11. doi:10.1038/nature03459.

Batista, S., Leclercq, L., Geroliminis, N., 2019. Estimation of regional trip length distributions for the calibration of the aggregated network traffic models. Transportation Research Part B: Methodological 122, $192-217$. URL: http://www.sciencedirect.com/science/article/ pii/S0191261518311603, doi:https://doi.org/10.1016/j.trb.2019.02.009.

Batista, S.F.A., Leclercq, L., Menendez, M., 2021a. Dynamic traffic assignment for regional networks with traffic-dependent trip lengths and regional paths. Transportation Research Part C: Emerging Technologies .

Batista, S.F.A., Seppecher, M., Leclercq, L., 2021b. Identification and characterizing of the prevailing paths on a urban network for mfd-based applications. Transportation Research Part C: Emerging Technologies .

Blondel, V., Decuyper, A., Krings, G., 2015. A survey of results on mobile phone datasets analysis. EPJ Data Science 4. doi:10.1140/epjds/ s13688-015-0046-0.

Calabrese, F., Di Lorenzo, G., Liu, L., Ratti, C., 2011. Estimating origin-destination flows using opportunistically collected mobile phone location data from one million users in boston metropolitan area. IEEE Pervasive Computing 10, 36-44.

Candia, J., González, M.C., Wang, P., Schoenharl, T., Madey, G., Barabási, A.L., 2008. Uncovering individual and collective human dynamics from mobile phone records. Journal of Physics A: Mathematical and Theoretical 41, 224015. URL: http://stacks . iop. org/1751-8121/ $41 / i=22 / a=224015$ ? key=crossref $.97 d 23 b 44 d e 724 a 7398482 c d 45 c 7 f e 01 a$, doi:10.1088/1751-8113/41/22/224015.

Castro, P.S., Zhang, D., Chen, C., Li, S., Pan, G., 2013. From taxi gps traces to social and community dynamics: A survey. ACM Comput. Surv. 46. URL: https://doi.org/10.1145/2543581.2543584, doi:10.1145/2543581.2543584.

Chen, C., Ma, J., Susilo, Y., Liu, Y., Wang, M., 2016. The promises of big data and small data for travel behavior (aka human mobility) analysis. Transportation Research Part C: Emerging Technologies .

Chen, G., Hoteit, S., Carneiro Viana, A., Fiore, M., Sarraute, C., 2018. Enriching sparse mobility information in call detail records. Computer Communications .

Chen, G., Viana, A.C., Fiore, M., Sarraute, C., 2019. Complete trajectory reconstruction from sparse mobile phone data. EPJ Data Science 8, 30. URL: https://doi.org/10.1140/epjds/s13688-019-0206-8, doi:10.1140/epjds/s13688-019-0206-8.

Cisco, 2020. Cisco Annual Internet Report (2018-2023). Technical Report. Cisco.

Çolak, S., Alexander, L.P., Alvim, B.G., Mehndiratta, S.R., González, M.C., 2015. Analyzing cell phone location data for urban travel: current methods, limitations, and opportunities. Transportation research record: Journal of the transportation research board 2526, 126-135.

Daganzo, C.F., 2007. Urban gridlock: Macroscopic modeling and mitigation approaches. Transportation Research Part B: Methodological 41, 49 62. URL: http://www.sciencedirect.com/science/article/pii/S0191261506000282, doi:https://doi.org/10.1016/j.trb. 2006.03 .001

Derrmann, T., Frank, R., Viti, F., Engel, T., 2017. Estimating urban road traffic states using mobile network signaling data, in: 2017 IEEE 20th International Conference on Intelligent Transportation Systems (ITSC), pp. 1-7. doi:10.1109/ITSC . 2017.8317718.

Gandica, Y., Carvalho, J., Sampaio dos Aidos, F., Lambiotte, R., Carletti, T., 2017. Stationarity of the inter-event power-law distributions. PLOS ONE 12,1-10. URL: https://doi.org/10.1371/journal.pone.0174509, doi:10.1371/journal.pone.0174509.

Geroliminis, N., Daganzo, C.F., 2008. Existence of urban-scale macroscopic fundamental diagrams: Some experimental findings. Transportation Research Part B: Methodological 42, 759 - 770. URL: http://www.sciencedirect.com/science/article/pii/S0191261508000180, doi:https://doi.org/10.1016/j.trb.2008.02.002.

Gonzalez, M.C., Hidalgo, C.A., Barabási, A.L., 2008. Understanding individual human mobility patterns. Nature 453, 779 EP -. URL: https : //doi.org/10.1038/nature06958.

Hoteit, S., Chen, G., Viana, A.C., Fiore, M.C., 2017. Spatio-Temporal Completion of Call Detail Records for Human Mobility Analysis, in: Rencontres Francophones sur la Conception de Protocoles, l'Évaluation de Performance et l'Expérimentation des Réseaux de Communication, Quiberon, France. URL: https://hal.archives-ouvertes.fr/hal-01516717.

Huang, H., Cheng, Y., Weibel, R., 2019. Transport mode detection based on mobile phone network data: A systematic review. Transportation Research Part C: Emerging Technologies 101, 297 - 312. URL: http://www.sciencedirect.com/science/article/pii/ S0968090X1831369X, doi:https://doi.org/10.1016/j.trc.2019.02.008.

Iqbal, M.S., Choudhury, C.F., Wang, P., González, M.C., 2014. Development of origin-destination matrices using mobile phone call data. Transportation Research Part C: Emerging Technologies 40, 63 - 74. URL: http://www.sciencedirect.com/science/article/pii/ S0968090X14000059, doi:https://doi.org/10.1016/j.trc.2014.01.002.

Janecek, A., Valerio, D., Hummel, K.A., Ricciato, F., Hlavacs, H., 2015. The cellular network as a sensor: From mobile phone data to real-time road traffic monitoring. IEEE TRANSACTIONS ON INTELLIGENT TRANSPORTATION SYSTEMS .

Jiang, S., Fiore, G.A., Yang, Y., Ferreira, J., Frazzoli, E., González, M.C., 2013. A review of urban computing for mobile phone traces: current methods, challenges and opportunities, in: UrbComp@KDD. 
Jurdak, R., Zhao, K., Liu, J., AbouJaoude, M., Cameron, M., Newth, D., 2015. Understanding human mobility from twitter. PLOS ONE 10 , 1-16. URL: https://doi.org/10.1371/journal.pone.0131469, doi:10.1371/journal.pone.0131469.

Leclercq, L., Chiabaut, N., Trinquier, B., 2014. Macroscopic fundamental diagrams: A cross-comparison of estimation methods. Transportation Research Part B: Methodological .

Lin, M., Hsu, W.J., 2014. Mining gps data for mobility patterns: A survey. Pervasive and Mobile Computing 12, 1-16. URL: https://www . sciencedirect.com/science/article/pii/S1574119213000825, doi:https://doi.org/10.1016/j.pmcj.2013.06.005.

Mariotte, G., Leclercq, L., Batista, S., Krug, J., Paipuri, M., 2020. Calibration and validation of multi-reservoir mfd models: A case study in lyon. Transportation Research Part B: Methodological 136, 62 - 86. URL: http://www.sciencedirect.com/science/article/pii/ S0191261519306769, doi:https://doi.org/10.1016/j.trb.2020.03.006.

Naboulsi, D., Fiore, M., Ribot, S., Stanica, R., 2016. Large-scale mobile traffic analy- sis: a survey. IEEE Communications Surveys Tutorials 18, $124-161$.

Nagle, A.S., Gayah, V.V., 2014. Accuracy of networkwide traffic states estimated from mobile probe data. Transportation Research Record 2421, 1-11. URL: https://doi.org/10.3141/2421-01, doi:10.3141/2421-01, arXiv:https://doi.org/10.3141/2421-01.

Osorio-Arjona, J., García-Palomares, J.C., 2019. Social media and urban mobility: Using twitter to calculate home-work travel matrices. Cities 89, 268 - 280. URL: http://www.sciencedirect.com/science/article/pii/S0264275118312976, doi:https://doi.org/10.1016/ j.cities.2019.03.006.

Ou, Q., Bertini, R.L., van Lint, J.W.C., Hoogendoorn, S.P., 2011. A theoretical framework for traffic speed estimation by fusing low-resolution probe vehicle data. IEEE Transactions on Intelligent Transportation Systems 12, 747-756. doi:10.1109/TITS.2011.2157688.

Paipuri, M., Barmpounakis, E., Geroliminis, N., Leclercq, L., 2021. Linear regression analysis of regional mean speed of athens city network using drone data: A multi-modal approach, in: 100th TRB Annual Meeting.

Ranjan, G., Zang, H., Zhang, Z.L., Bolot, J., 2012. Are call detail records biased for sampling human mobility? ACM SIGMOBILE Mobile Computing and Communications Review 16, 33-44. doi:10.1145/2412096.2412101.

Shang, J., Zheng, Y., Tong, W., Chang, E., Yu, Y., 2014. Inferring gas consumption and pollution emissions of vehicles throughout a city. Proceedings of the ACM SIGKDD International Conference on Knowledge Discovery and Data Mining .

Toch, E., Lerner, B., Ben-Zion, E., Ben-Gal, I., 2018. Analyzing large-scale human mobility data: a survey of machine learning methods and applications. Knowledge and Information Systems URL: https://doi.org/10.1007/s10115-018-1186-x, doi:10.1007/ s10115-018-1186-x.

Toole, J.L., Çolak, S., Sturt, B., Alexander, L.P., Evsukoff, A., González, M.C., 2015. The path most traveled: Travel demand estimation using big data resources. Transportation Research Part C: Emerging Technologies 58, 162 - 177. URL: http://www.sciencedirect.com/science/ article/pii/S0968090X15001631, doi:https://doi.org/10.1016/j.trc.2015.04.022. big Data in Transportation and Traffic Engineering.

Yildirimoglu, M., Geroliminis, N., 2014. Approximating dynamic equilibrium conditions with macroscopic fundamental diagrams. Transportation Research Part B: Methodological 70, 186 - 200. URL: http://www.sciencedirect.com/science/article/pii/S0191261514001568, doi:https://doi.org/10.1016/j.trb.2014.09.002.

Zhan, X., Zheng, Y., Yi, X., Ukkusuri, S.V., 2017. Citywide traffic volume estimation using trajectory data. IEEE Transactions on Knowledge and Data Engineering 29, 272-285. doi:10.1109/TKDE.2016.2621104.

Zhang, J., Wang, K., Lin, W.H., Xu, X., Chen, C., 2011. Data-driven intelligent transportation systems: A survey. IEEE Transactions on Intelligent Transportation Systems 12, 1624-1639. doi:10.1109/TITS.2011.2158001.

Zheng, Y., Liu, F., Hsieh, H.P., 2013. U-air: When urban air quality inference meets big data. URL: https://www.microsoft.com/en-us/ research/publication/u-air-when-urban-air-quality-inference-meets-big-data/. 